\title{
Inertial flow past a finite-length axisymmetric cylinder of aspect ratio 3: effect of the yaw angle
}

\author{
Jean-Lou Pierson and Abdelkader Hammouti \\ IFP Energies nouvelles, 69360 Solaize, France* \\ Franck Auguste \\ IMFT, Université de Toulouse, 31400 Toulouse, France \\ CERFACS, 31057 Toulouse, France \\ Anthony Wachs \\ Department of Mathematics, University of British Columbia, Vancouver, BC, Canada, \\ Department of Chemical $\&$ Biological Engineering, \\ University of British Columbia, Vancouver, BC, Canada
}

(Dated: July 26, 2018) 


\begin{abstract}
We study the flow past a finite-length yawed 3D cylinder by a Finite Volume / Fictitious Domain $(\mathrm{FV} / \mathrm{FD})$ method developed in [63]. We validate our non-boundary-fitted method against boundary-fitted numerical results for a finite-length cylinder whose axis is parallel to the streamwise direction. Drag and lift forces exerted on the cylinder and vortex shedding onset and frequency are carefully analysed. Satisfactory agreement with published results give strong confidence in the numerical methodology provided the boundary layer is accurately resolved. Then, we carry out a detailed study of the flow past a yawed cylinder of aspect ratio $L / D=3$ (where $L$ is the cylinder length and $D$ is the cylinder diameter) at moderate Reynolds numbers $(25 \leqslant R e \leqslant 250)$. We show that the wake pattern depends strongly on $R e$ and the yaw angle $\theta$ with respect to the streamwise direction. Various regimes are encountered including standing-eddy pattern, steady shedding of one and two pairs of counter-rotating vortices, periodic shedding of two pairs of counter-rotating vortices and unsteady shedding of hairpin shaped vortices. The standing-eddy pattern regime shows different forms of behaviour and symmetry as function of $\theta$. Hydrodynamic forces exerted on the cylinder are well approximated by laws derived in the Stokes flow regime (benefitting from the linearity of the equations), even for moderate Reynolds numbers. This result is in agreement with recent findings of Sanjeevi and Padding [53] who studied the flow past spheroidal particles. For the highest Reynolds numbers $(R e=150,200,250)$ we show that simple force laws can be derived from simple geometrical assumptions. These simple laws yield a satisfactory match with our numerical results.
\end{abstract}

\title{
Keywords: fictitious domain method, finite-length cylinder, hydrodynamic force/torque, wake instability
}




\section{INTRODUCTION}

Fluidized beds are frequently encountered in various industrial processes such as catalysis and biomass gasification. For computational reasons, Euler-Lagrange and Euler-Euler methods are usually preferred to direct numerical simulation to tackle this kind of problem. The former class of methods has been applied with success to spouted beds and bubbling beds of spherical particles $[6,7]$. However the averaging procedure used to derive the Euler-Lagrange equations yields more unknowns than equations [26]. Closure laws for the hydrodynamic force and torque exerted on solid bodies are thus needed to solve the problem. While a large number of studies exist in the literature on the flow past a single or multiple spherical particles (see for instance Clift et al. [9] among many others), little is known about finitelength cylindrical particles that are frequently used in bubbling fluidized beds. Therefore, we investigate the flow past a single finite-length yawed cylinder using numerical simulation to understand the hydrodynamic force and torque exerted on it as a step towards the understanding of the hydrodynamic force and torque exerted on many finite-length (presumably randomly) yawed cylinders.

One of the earliest studies of the flow past a cylinder oriented perpendicularly to the streamwise direction is due to Wieselsberger [64]. Both infinite-length and finite-length cylinders were considered. The aspect ratio is defined as $L / D$, where $L$ is the cylinder length and $D$ the cylinder diameter. Wieselsberger [64] covered a large range of Reynolds numbers $R e=\rho D U / \mu$ from 400 to $8 \times 10^{5}$ where $\mu, \rho$ and $U$ are the dynamic viscosity, the density and the far field inlet velocity, respectively. Wieselsberger [64] observed that the drag coefficient decreases when decreasing $L / D$. Zdravkovich et al. [67] studied the flow past a perpendicular cylinder of finite aspect ratio $1 \leq L / D \leq 10$ at high Reynolds numbers $6 \times 10^{4} \leq R e \leq 2.6 \times 10^{5}$. The drag coefficient was also observed to decrease when decreasing $L / D$. Zdravkovich et al. [67] observed a kind of vortex shedding in the range $2 \leq L / D \leq 8$ and an asymmetric flow pattern for $1 \leq L / D \leq 3$ for all values of $R e$ he investigated. Inoue and Sakuragi [25] performed a detailed numerical study of the flow past a finite-length cylinder. The selected aspect ratios and Reynolds numbers were respectively $0.5 \leq L / D \leq 100$ and $40 \leq R e \leq 300$. They identified five different vortex shedding patterns depending on both aspect ratio and Reynolds number. They also showed that the critical Reynolds number for the onset of the unsteady regime decreases with $L / D$. 
The flow past an infinite yawed cylinder has also been widely studied in relation to its numerous industrial applications as, e.g., offshore risers in oil industry or strand wires of floating offshore wind turbines. Relf and Powell [48] studied experimentally the flow of air past strand wires at $R e \approx 10000$. In this regime they observed that the normal force exerted on the cylinder is proportional to the square of the normal component of the velocity (the normal direction here is given by the normal vector to the cylinder surface in the plane defined by the cylinder axis and the far field inlet velocity). Sears [54] theoretically demonstrated, using boundary layer theory, that the flow past an infinite-length yawed cylinder is determined by the normal component of the velocity. In other words, the force on an infinite-length cylinder tilted by an angle $\theta$ with respect to the flow direction is identical to the force on the same infinite-length cylinder in cross-flow with velocity $U \sin \theta$. This law called independence principle (IP) has been widely used to predict the hydrodynamic force exerted a yawed cylinder. However this principle suffers from limitations summarized in Zdravkovich [68, p 955] and primarily due to simplifications inherent to its boundary layer derivation. Moreover its application to yawed cylinders of finite aspect ratio $L / D$ remains an open question.

Studies of the flow past a finite-length yawed or aligned cylinder (aligned in the sense that its symmetry axis is parallel to the streamwise direction) are more sparse compared to the large amount of works on the flow past a cylinder perpendicular to the streamwise direction. Auguste [2] and Auguste et al. [3] numerically studied the wake past an aligned cylinder of small aspect ratio $0 \leq L / D \leq 1$. The Reynolds number selected was in the range $0 \leq R e \leq 400$. Auguste [2] observed that the critical Reynolds number for the transition to the unsteady regime varies strongly with the aspect ratio $L / D$. To the authors' knowledge, the bifurcation scenario for $L / D>1$ has not been studied so far. Recently Chrust et al. [8] evidenced the effect of $L / D$ on the wake past aligned spheroids. Ramberg [47] studied experimentally the flow past free-ended yawed cylinders and yawed cylinders fitted with endplates at Reynolds number $160 \leq R e \leq 1100$. He showed that results were very sensitive to the cylinder end conditions. Recently Vakil and Green [59] performed a complete analysis based on numerical results of the flow past a yawed cylinder of aspect ratio $2 \leq L / D \leq 20$ for moderate Reynolds number $1 \leq R e \leq 40$. They proposed empirical relations for the drag force and the lift force exerted on the cylinder. They also checked the validity of the IP. Even if the range of Reynolds number studied was lower than the one required for the 
strict application of the boundary layer theory, they obtained a relatively good agreement between the values of the drag predicted by the IP and the actual values of the drag for large $\theta>45^{\circ}$.

A large number of numerical studies on the flow past a solid obstacle, regardless of its shape and angular position, use a boundary-fitted method in order to optimally compute the flow in the boundary layer around that obstacle $[2,25,59]$. These methods are very accurate but not designed to efficiently handle (i) multiple angular positions of a single solid obstacle of arbitrary shape, (ii) a large number of solid obstacles of arbitrary shape, or (ii) a single/multiple freely moving rigid bodies. In all these 3 cases, extensive re-meshing is required, either as a function of the solid obstacle angular position [59] or as a function of the time dependent position of the particles [23]. For the 3 aforementioned flow configurations, fictitious domain methods are usually preferred. Indeed, the no-slip boundary condition on the particle surface and/or the rigid body motion inside the particle volume are imposed on a fixed Eulerian grid using forcing terms added to the fluid momentum conservation equation [35]. These methods have proven to be efficient for the flow past multiple rigid obstacles and the free motion of multiple rigid particles, with a spherical, spheroidal and angular shape (see Ardekani et al. [1], Uhlmann and Dušek [58], Wachs et al. [63] among many others).

The investigation reported here, based on about a hundred simulations spanning inclination angles $\theta$ from $0^{\circ}$ to $90^{\circ}$ and Reynolds numbers from 25 to 250, provides the first description of the flow past short yawed cylinders of aspect ratio 3. The selected aspect ratio and Reynolds number ranges are particularly relevant to many chemical engineering applications. In fact, cylindrical pellets with an aspect ratio around 3 are frequently encountered in fixed and fluidized bed reactors. The paper is organized as follows. We present the governing equations and the numerical procedures in Section. We end this section with a careful validation of our numerical method by comparing our computed results to the boundary-fitted results summarized in Appendix. We describe and analyze the flow past a $L / D=3$ yawed cylinder in inertial regimes in Section. A special attention is paid to the transition between wake regimes as a function of $R e$ and $\theta$. In Section, we attempt to find simple laws for the drag, lift and torque coefficients in order to provide closure laws needed by Euler-Lagrange methods. In particular the validity of the IP as models derived in the Stokes regimes are discussed. Main conclusions and future work are presented in Section . 


\section{NUMERICAL PROCEDURES AND VALIDATION}

Computations are performed with our Finite Volume / Fictitious Domain method implemented in our parallel code PeliGRIFF $[62,63]$. We have used this method and corresponding code in many flow configurations, including fixed obstacles and freely-moving particles, with spherical and non-spherical particles, and with or without heat transfer. For the sake of completeness, we shortly summarize in this section the main features of our fictitious domain method. The whole numerical method follows almost exactly what we described in Dorai et al. [13]. The last subsection is dedicated to a detailed validation of the code in the flow configuration we are interested in here, i.e., the inertial flow past a single cylinder in an unbounded domain.

\section{Solution algorithm}

The three-dimensional unsteady incompressible Navier-Stokes equations with appropriate initial conditions and boundary conditions subject to the rigid body motion (motionless in the particular case of the flow problem examined in this paper) constraint in the region occupied by the cylinder denoted $P$ (and filled with fictitious fluid) are solved by a first-order Marchuk-Yanenko time splitting solution algorithm. We use a simple two-step splitting: at each discrete time, we (i) solve the incompressible Navier-Stokes equations and then (ii) solve the fictitious domain problem to enforce the rigid body motion constraint. Problem (i) is solved with a classical $L^{2}$-projection scheme while Problem (ii) is a saddle-point problem solved by a Uzawa/conjugate gradient algorithm [19,63]. While the solution of Problem (i) is second-order accurate in time, the whole solution algorithm is first-order accurate in time only due to the first-order Marchuk-Yanenko splitting. Please note that the presence of the explicit forcing term $\pm \mathbf{f}^{n}$ on the right-hand side of (1a) and (2a) significantly enhances the coupling of the two sub-problems [63]. $\mathbf{f}$ is simply a smoothed equivalent of the distributed Lagrange multiplier $\boldsymbol{\lambda}^{n}$ associated to the constraint $\mathbf{u}=\mathbf{0}$ in $P$ [63]. At each discrete time $t^{n+1}$ where $n$ refers to the discrete time index, we know the fluid velocity $\mathbf{u}^{n}$, the pressure $p^{n}$ and the distributed Lagrange multiplier $\boldsymbol{\lambda}^{n}$ in $P$, and solve the following equations for $\mathbf{u}^{n+1}, p^{n+1}$ and $\boldsymbol{\lambda}^{n+1}$ as follows:

- A classical $L^{2}$-projection scheme for the solution of the Navier- Stokes problem: find 
$\mathbf{u}^{n+1 / 2}$ and $p^{n+1}$ such that

$$
\begin{aligned}
& \rho \frac{\tilde{\mathbf{u}}^{n+1 / 2}-\mathbf{u}^{n}}{\Delta t}-\frac{\mu}{2} \nabla^{2} \tilde{\mathbf{u}}^{n+1 / 2}=-\nabla p^{n}+ \\
& \frac{\mu}{2} \nabla^{2} \mathbf{u}^{n}-\frac{\rho}{2}\left(3 \mathbf{u}^{n} \cdot \nabla \mathbf{u}^{n}-\mathbf{u}^{n-1} \cdot \nabla \mathbf{u}^{n-1}\right)-\mathbf{f}^{n}, \\
& \nabla^{2} \psi^{n+1}=\frac{\rho}{\Delta t} \nabla \cdot \tilde{\mathbf{u}}^{n+1 / 2}, \\
& \mathbf{u}^{n+1 / 2}=\tilde{\mathbf{u}}^{n+1 / 2}-\frac{\Delta t}{\rho} \nabla \psi^{n+1}, \\
& p^{n+1}=p^{n}+\psi^{n+1}-\frac{1}{2} \frac{\mu \Delta t}{\rho} \nabla^{2} \psi^{n+1}
\end{aligned}
$$

where $\rho$ denotes the fluid density, $\mu$ the viscosity and $\psi^{n+1}$ the auxiliary potential. In (1a), the advection and viscous terms are discretized in time by an Adams-Bashford scheme and a Crank-Nicholson scheme, respectively.

- A fictitious domain problem: find $\mathbf{u}^{n+1}$ and $\boldsymbol{\lambda}^{n+1}$ such that

$$
\begin{aligned}
\rho \frac{\mathbf{u}^{n+1}-\mathbf{u}^{n+1 / 2}}{\Delta t}+\boldsymbol{\lambda}^{n+1} & =\mathbf{f}^{n}, \\
\mathbf{u}^{n+1} & =\mathbf{0} \text { in } P
\end{aligned}
$$

It can easily be shown [46] that the hydrodynamic force $\mathbf{F}$ and torque $\mathbf{T}$ exerted on the cylinder can be expressed as volume integrals over the cylinder $P$ of the distributed Lagrange multiplier $\boldsymbol{\lambda}$ as follows:

$$
\begin{aligned}
& \mathbf{F}=\int_{S} \boldsymbol{\sigma} \cdot \mathbf{n} d S=\int_{P} \boldsymbol{\lambda} d V \\
& \mathbf{T}=\int_{S} \mathbf{r} \times \boldsymbol{\sigma} \cdot \mathbf{n} d S=\int_{P} \mathbf{r} \times \boldsymbol{\lambda} d V
\end{aligned}
$$

where $\boldsymbol{\sigma}=-p \boldsymbol{I}+2 \mu \boldsymbol{e}$ is the Newtonian stress tensor, $\boldsymbol{I}$ the identity tensor, $\boldsymbol{e}=\frac{1}{2}\left(\nabla \mathbf{u}+\nabla \mathbf{u}^{t}\right)$ the strain-rate tensor, $\nabla \mathbf{u}$ the velocity gradient tensor, $\mathbf{r}$ the position vector relative to the solid mass center and $\mathbf{n}$ the unit vector normal to the cylinder surface $S$.

\section{Space discretization scheme}

(1a)-(1d) are solved on a cartesian grid with a Finite Volume approach. The pressure is cell-centered while the velocity component $i$ is located at the cell face perpendicular to direction $i$. A second order central discretization scheme is employed for the diffusion term 
while the convective term is treated with a total variation diminishing (TVD) scheme and a Superbee flux limiter. Without any immersed solid body in the flow domain, the overall space accuracy of the fluid discretization scheme is 2nd order. However due to the presence of the immersed boundary solid bodies, the method is not fully second order in space [63].

In order to enforce the zero velocity constraint inside the cylinder, we use a collocation point method in which the basis functions for the distributed Lagrange multiplier are delta Dirac functions $[19,63]$. A set of Lagrangian points is distributed along the surface and inside the cylinder. Interior points are distributed on the staggered grid at the location of velocity points. Distributing points uniformly along the cylinder surface, called boundary points, is much more challenging. The detailed method developed in the PeliGRIFF code is described in Appendix. To summarize, we divide the cylinder in two main areas : its length and its two end disks. The surface area defined along the cylinder length is mapped using a diamondshaped mesh while the two end disks are mapped with a specific spiral distribution. This methodology ensures that boundary points are close to uniformly distributed. Regardless of the body shape, the quasi uniform distribution of boundary points property has proven to be important for the accurary of the computed solution [63]. Finally, a multi-dimensional 2nd order quadratic reconstruction operator is used to enforce the zero velocity constraint at the Lagrangian points located on the cylinder surface. The construction of the 3D stencil of this operator relies on the orientation of the outward normal vector to the particle boundary and is a key ingredient of the space discretization scheme. It has also proven to significantly improve the accuracy of the computed solution. The accuracy of our scheme with immersed solid bodies is between first and second order, depending on the flow configuration, but the main asset of the multi-dimensional 2nd order quadratic reconstruction operator is that it does not require any hydrodynamic radius calibration neither for spheres nor for any other shape [63], and can hence be safely applied to a cylinder here.

The explicit forcing term $\mathbf{f}^{n}$ in (1a) and (2a) is computed using the multi-dimensional 2nd order quadratic reconstruction operator for boundary points and is smoothed out for interior points using a simple hat function defined on a support that spans three grid points in each direction. This simple procedure has proven to be efficient in all our previous studies (see Dorai et al. [13], Rahmani and Wachs [46], Wachs et al. [63] among others) and shares some similarities with the regularized Dirac delta function used by Uhlmann [57] and Kempe and Fröhlich [29], although in these works a regularized Dirac delta function is employed 
in an IBM fashion to enforce the no-slip boundary condition on boundary points only, leaving interior points free to flow as a fluid. In our method, since the distributed Lagrange multipliers $\boldsymbol{\lambda}^{n+1}$ that implicity enforces the zero velocity contraint in the cylinder use actual Dirac delta functions as basis functions, the a posteriori computation of the hydrodynamic force $\mathbf{F}$ and torque $\mathbf{T}$ exerted on the cylinder is rather straightforward. In fact, computing $\mathbf{F}$ and $\mathbf{T}$ at time $t^{n+1}$ translates into summing the distributed Lagrange multipliers as:

$$
\begin{aligned}
\mathbf{F}^{n+1} & =\int_{P} \boldsymbol{\lambda}^{n+1} d V=\sum_{j=0}^{N_{i}-1} \boldsymbol{\lambda}_{j}^{n+1}+\sum_{k=0}^{N_{b}-1} \boldsymbol{\lambda}_{k}^{n+1} \\
\mathbf{T}^{n+1} & =\int_{P} \mathbf{r} \times \boldsymbol{\lambda}^{n+1} d V=\sum_{j=0}^{N_{i}-1} \mathbf{r}_{j} \times \boldsymbol{\lambda}_{j}^{n+1}+\sum_{k=0}^{N_{b}-1} \mathbf{r}_{k} \times \boldsymbol{\lambda}_{i}^{n+1}
\end{aligned}
$$

where $N_{i}$ and $N_{b}$ denote the number of interior points and number of boundary points, respectively.

\section{Computational domain}

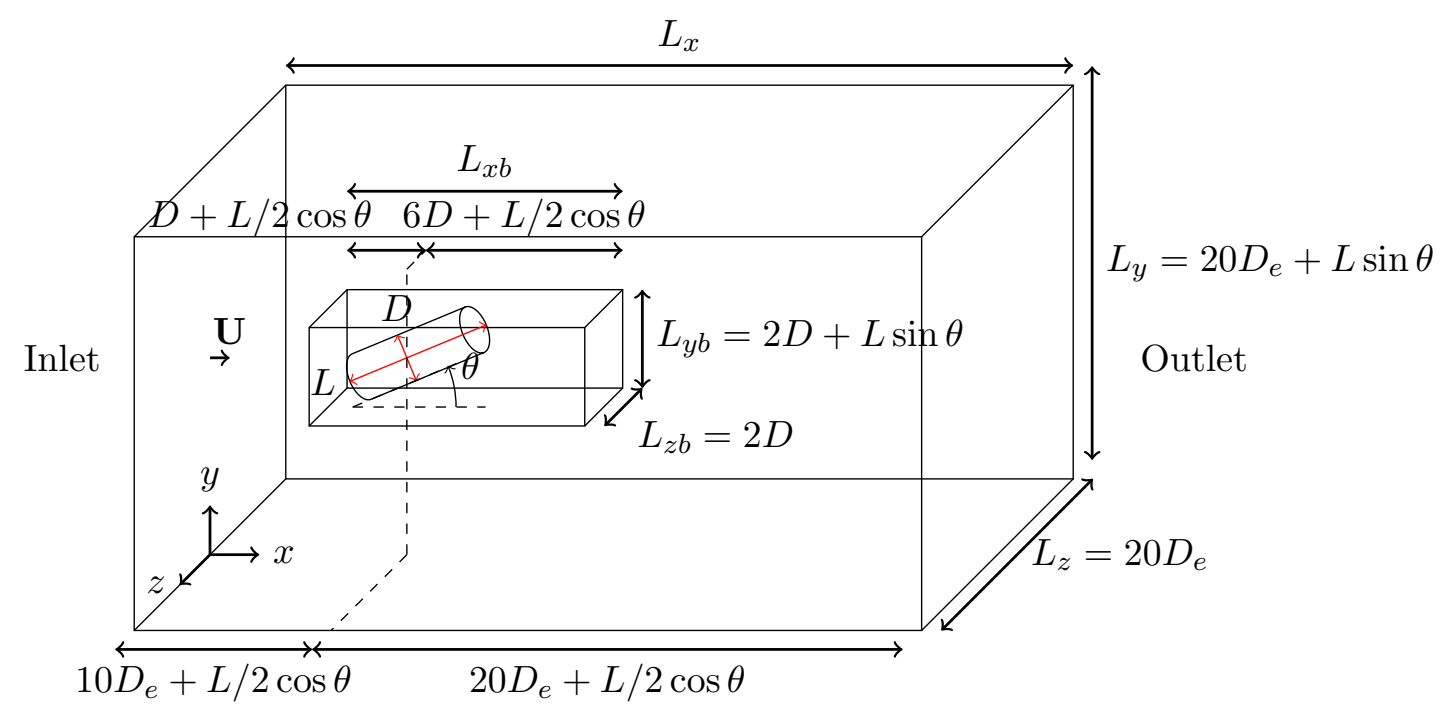

FIG. 1: Sketch of the computational domain.

The design of a computational flow domain relevant of an unbounded domain and valid in all studied configurations (various aspect ratios and yawed angles) while keeping its size "not too large" is not straightforward. In the following we briefly review several computational domains used in the past literature. Auguste [2] selected a cylindrical domain whose length 
and radius are $25 D$ and $10 D$, respectively, where $D$ is the disk diameter. The author focused on the flow past various disks of aspect ratio varying from 0 to 1 . Special attention is paid to the distance between the disk and the outlet boundary which has to be at least $15 D$ to avoid errors on the computation of the hydrodynamic force. Inoue and Sakuragi [25] studied the flow past cylinders perpendicular to the streamwise direction. In their study, the aspect ratio varied from 0.5 to 100 . Their computational domain is a box and the authors defined five computational domains depending on the range of aspect ratio studied. In particular the length of the domains in the streamwise direction ranges from $115 D$ to $190 D$. The height of the domains, i.e., the domain size in the direction parallel to the cylinder axis, varies linearly with $L$ as $L+60 D$. The depth of the domains, i.e., the domain size in the direction perpendicular to both the cylinder axis and the streamwise direction, is equal to $60 D$ and is thus fixed for all aspect ratios studied. Vakil and Green [59] studied the flow past a yawed cylinder of variable aspect ratio ranging from 2 to 20. Their computational domain shares some similarities with the computational domain selected by Inoue and Sakuragi [25]. Indeed both the domain length and the domain height depend on $L$ and are set to $25 L$ and $12 L$, respectively, while the domain depth is fixed and set to $50 \mathrm{D}$.

In this work, we define the size of our domain using a length proportional to the equivalent spherical diameter (the diameter of a sphere with same volume as the cylinder): $D_{e}=$ $\left(L D^{2}\right)^{1 / 3}$. This choice ensures that the domain size changes with the size of the cylinder while remaining relatively small. This design rule remains valid at least up to $L / D=10$ as discussed later in this section.

Simulations are performed in a cuboid domain (i.e., a box) meshed with an irregular cartesian grid. $(x, y, z)$ coordinates are defined as follows: $x$ is parallel to the streamwise direction, $y$ is perpendicular to the streamwise direction in the plane defined by the streamwise direction and cylinder axis, $z$ is perpendicular to the plane defined by the streamwise direction and cylinder axis. The box size is adapted to the cylinder diameter $D$, to the cylinder length $L$ and the angular position of the cylinder defined by the angle $\theta$ between the cylinder axis and the streamwise direction. Indeed the length $L_{x}$, height $L_{y}$ and depth $L_{z}$ of the box are respectively $30 D_{e}+L \cos \theta, 20 D_{e}+L / 2 \cos \theta$ and $20 D_{e}$ (see FIG. 1). $L_{y}$ and $L_{z}$ are chosen sufficiently large to avoid wall effects for moderate Reynolds numbers. $L_{z}$ is defined in such a way that the wake can develop without being perturbed by the outlet boundary. The domain is divided into two main regions. An inner region around the 
cylinder which is made of regular cells. The size of this subdomain $\left(L_{x b}, L_{y b}, L_{z b}\right)$ is specified in FIG. 1. $L_{x b}$ is larger downstream of the cylinder to ensure that the near wake is well captured. The outer region is made of stretched cells of growing size away from the cylinder that smoothly match the inner region cell size.

Boundary conditions are prescribed as follows. Symmetry boundary conditions are imposed on the 4 lateral walls. At the inlet a uniform velocity profile is imposed as $\mathbf{u}=(U, 0,0)$. The imposition of the outlet boundary condition is not straightforward and different choices can be found in the literature [45, p. 36]. The choice made in the PeliGRIFF code is to impose a zero velocity gradient condition $\partial \mathbf{u} / \partial n=0$ and an arbitrary reference pressure $p=p_{\text {ref }}$ at the outlet boundary. Such a boundary condition has been used with success by other authors as, e.g., Kim and Elghobashi [31] to study the unsteady flow past a sphere.

In the following sections, the numerical method described above is applied to the study of the flow past a finite-length yawed cylinder. The Reynolds number $R e=\rho U D / \mu$, the yaw angle $\theta$ and the aspect ratio $L / D$ fully characterize the system. Governing equations and flow variables are non-dimensionalized by introducing the following scales: $D$ for length, $U$ for velocity, $D / U$ for time (implying that we employ a convective time scale), $\rho U^{2}$ for pressure, $\rho U^{2} / D$ for Lagrange multiplier and $U / D$ for vorticity. The choice of the length scale is far from straightforward. Indeed assorted definitions are used in the literature : Sears [54] used the cylinder length, Vakil and Green [59] used the cylinder diameter and Hölzer and Sommerfeld [22] used the equivalent diameter. The analysis to come involves the following dimensionless output parameters:

- The Strouhal number $S t=f D / U$ when the wake and the force experienced by the body are unsteady and periodic. St compares the vortex shedding frequency $f$ to the flow characteristic frequency $U / D$. Different ways of measuring $f$ are found in the literature : local measurement of the oscillation of variables in the wake [25] or measurement of the oscillation of the lift force [2]. We use the latter definition. When there is no ambiguity on the orientation of the lift force (i.e., when the wake exhibits a symmetry plane), we do not specify how we measure St. Otherwise, when the wake is fully three-dimensional and/or oscillates with multiple frequencies, we elaborate on how $S t$ is measured.

- To characterize the hydrodynamic force and torque exerted on the cylinder, we define 
the classical drag coefficient as $C_{D}=F_{x} /\left(1 / 2 \rho U^{2} L D\right)$ and lift coefficients as $C_{L i}=$ $F_{i} /\left(1 / 2 \rho U^{2} L D\right), i=(y, z)$. Those coefficients represent ratios of hydrodynamic force components to an inertial characteristic force $1 / 2 \rho U^{2} L D$. $L D$ is proportional to the lateral surface area of the cylinder. It is common practice to define the drag coefficient of a bluff body using the surface area of the projection of the body on a plane normal to the streamwise direction [5, p 339]. Our choice to use $L D$ as the reference surface area for drag and lift coefficients is guided by two main reasons: (i) when $L \gg D$ and $\theta>0$ the projected surface area of the disk becomes negligible compared to the lateral surface area of the cylinder, and (ii) since the reference surface area does not depend on $\theta$, comparison between hydrodynamic force and torque at different yaw angles is made simpler. Accordingly, the torque coefficient $C_{T}$ is defined as $C_{T}=T /\left(1 / 2 \rho U^{2} L^{2} D\right)$.

- The wake is visualized using the $Q$ criterion [24] defined as follows:

$$
Q=\frac{1}{2}\left(e_{i j} e_{i j}-\omega_{i j} \omega_{i j}\right)
$$

where $e_{i j}=1 / 2\left(\partial u_{i} / \partial x_{j}+\partial u_{j} / \partial x_{i}\right)$ and $\omega_{i j}=1 / 2\left(\partial u_{i} / \partial x_{j}-\partial u_{j} / \partial x_{i}\right)$ are the components of the strain-rate and vorticity tensors, respectively.

In all computations the dimensionless time step is set to $\Delta t=1.25 \times 10^{-2}$ in order to (i) satisfy the CFL condition and (ii) make the splitting error tolerable. Each simulation is run over a dimensionless time interval at least equal to $t=100$. When the flow is unsteady and periodic, simulations are run until the system has experienced at least six periods of oscillation. Time-averaged quantities are denoted with an overline symbol, i.e., time-averaged value of $x$ is denoted $\bar{x}$.

In order to evaluate the influence of the domain size on the numerical results, the flow past a $\left(L / D=10, \theta=90^{\circ}\right)$ cylinder at $R e=100$ is computed with 2 different domain sizes: (i) the domain as defined in FIG. 1 and (ii) a domain 1.5 larger in all directions. We keep the same grid size in the inner region around the cylinder for the 2 domains. The selected grid size corresponds to 32 grid points over the cylinder diameter. This space resolution is enough at $R e=100$ to capture well the viscous boundary layer that develops along the cylinder surface. Results obtained with the two domains are compared to each other and to those of Inoue and Sakuragi [25]. TAB. I shows that the difference between our 2 domains is less than $1 \%$ for both the drag coefficient $C_{D}$ and the Strouhal number St. Our computed 


\begin{tabular}{crc}
\hline Domain & $C_{D}$ & $S t$ \\
\hline Medium & 3.753 & 0.1280 \\
Large & 3.789 & 0.1289 \\
Inoue and Sakuragi $[25]$ & - & 0.1235 \\
\hline
\end{tabular}

TABLE I: Drag coefficient $C_{D}=F_{x} /\left(1 / 2 \rho U^{2} L D\right)$ and Strouhal number $S t=f D / U$ for two domain sizes. The medium domain is the one shown in FIG. 1. The large domain is

1.5 larger in all directions. Data of Inoue and Sakuragi [25] are taken as reference.

St matches very well St computed by Inoue and Sakuragi [25], the relative error being about to $3.5 \%$. The domain size as defined in Fig. 1 is thus used for all computations presented in this paper.

Flow past a $L / D=1$ cylinder with $\theta=0$ : comparison with boundary fitted mesh results

In this section we compare our results to results presented in Appendix obtained with a boundary-fitted method implemented in the JADIM code from IMFT that enables one to adopt an arbitrarily fine body-fitted mesh in the boundary layer around the cylinder. The JADIM numerical solution is hence deemed to be a reference solution. A cylinder of aspect ratio $L / D=1$ aligned with the streamwise direction is considered. To demonstrate the ability of our approach to properly describe the flow past a yawed cylinder, we select 5 Reynolds numbers $R e=25,50,100,200,360$ covering both the stationary regime with axial symmetry and the unsteady vortex shedding regime.

TAB. II shows $C_{D}$ and the recirculation length $l_{r}$ for $R e=25,50,100,200$ (stationary regime) as a function of grid size. $l_{r}$ is measured from the downstream tip of the cylinder to the downstream tip of the eddy. The downstream tip of the eddy is fitted with a fourth-order polynomial. The error on $C_{D}$ using the coarsest grid (16 grid points per cylinder diameter) is less than $3.5 \%$ except for $R e=200$. Since the thickness of the boundary layer scales as $\mathcal{O}\left(D / R e^{1 / 2}\right)$, there is approximatively one grid point in the viscous boundary layer at $R e=200$. This is far from being sufficient, since even for boundary fitted mesh about 3 to 5 five grid points are necessary to accurately describe the viscous boundary layer [2]. 


\begin{tabular}{rccccc}
\hline & & $C_{D}$ & $E\left(C_{D}\right) \%$ & $l_{r}$ & $E\left(l_{r}\right) \%$ \\
\hline$R e=25$ & Auguste [2] & 9.2868 & - & 0.430 & \\
& 16 cells $/ D$ & 9.4441 & 1.6933 & 0.453 & 5.35 \\
& 32 cells $/ D$ & 9.3591 & 0.77811 & 0.447 & 3.84 \\
\hline$R e=50$ & Auguste [2] & 6.1591 & - & 0.720 & - \\
& 16 cells $/ D$ & 6.2668 & 1.7486 & 0.741 & 2.99 \\
& 32 cells $/ D$ & 6.2034 & 0.72013 & 0.732 & 1.67 \\
\hline$R e=100$ Auguste [2] & 4.2210 & - & 1.12 & - \\
16 cells $/ D$ & 4.3533 & 3.1338 & 1.17 & 4.02 \\
32 cells $/ D$ & 4.2532 & 0.76104 & 1.14 & 1.43 \\
\hline$R e=200$ Auguste $[2]$ & 2.9468 & - & 1.630 & - \\
16 cells $/ D$ & 3.2466 & 10.173 & 1.86 & 14.1 \\
32 cells $/ D$ & 3.0324 & 2.9033 & 1.68 & 2.79 \\
\hline
\end{tabular}

TABLE II: Comparison of the drag coefficient $C_{D}$ and length of standing eddy $l_{r}$ given by our numerical method and the boundary-fitted method used in Appendix for $25 \leq R e \leq 200$. The number of cells distributed along the cylinder diameter varies from 16 to 32 . $E\left(C_{D}\right)$ and $E\left(l_{r}\right)$ represent the relative error on the drag coefficient and the relative error on the length of the standing eddy, respectively.

The error on $l_{r}$ using the coarsest grid is larger than $3 \%$ for all Reynolds numbers. This error decreases significantly using a twice finer grid (32 grid points per cylinder diameter). However, we note that the error made on $l_{r}$ is still higher than $3 \%$ for $R e=25$. The increase of numerical errors for low Reynolds number flows is pointed out by Wachs et al. [63] [Note1] and is often associated to the operator splitting error that scales as $\Delta t / R e[41]$.

FIG. 2 shows the vortex shedding behind the cylinder at $R e=360$. The wake exhibits a planar symmetry in the $(x, y)$ cut plane containing the cylinder axis. Hairpin vortices are shed periodically behind the cylinder. This type of wake is a distinctive feature of wake instability and has been observed in the literature for the flow past a sphere [52], the flow past a cylinder [25] and even when a sphere crosses a fluid-fluid interface [42]. The vortex structure is single-sided, i.e., hairpin vortices always rotate in the same direction. Moreover, vortices are all shed with the same orientation which induces an averaged non-zero lift force 


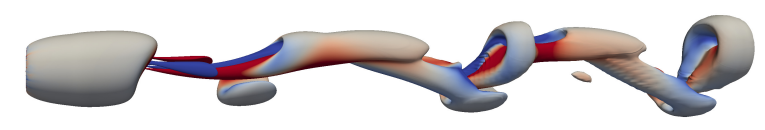

FIG. 2: Wake pattern past a $L / D=1$ cylinder aligned with the streamwise direction at $R e=360.96$ cells are distributed along the cylinder diameter. The wake is visualized using the $Q$ criterion. Isosurfaces of $Q=10^{-3}$ are shown and are coloured by the horizontal vorticity ranging from -0.2 to 0.2 .

on the cylinder (the direction of the lift force is defined unambiguously in that case owing to the wake symmetry plane).

\begin{tabular}{rcccc}
\hline & $\overline{C_{D}}$ & $E\left(\overline{C_{D}}\right) \%$ & $S t$ & $E(S t) \%$ \\
\hline$R e=360$ Auguste [2] & 0.578 & - & 0.118 & - \\
16 cells $/ D$ & 0.808 & 39.8 & - & - \\
32 cells $/ D$ & 0.678 & 17.3 & 0.124 & 4.67 \\
64 cells $/ D$ & 0.609 & 5.44 & 0.118 & 0.113 \\
96 cells $/ D$ & 0.597 & 3.36 & 0.117 & 0.762 \\
\hline
\end{tabular}

TABLE III: Comparison of the drag coefficient $C_{D}$ and Strouhal number $S t$ given by our numerical method and the boundary-fitted method used in Appendix for $R e=360$. The number of cells distributed along the cylinder diameter varies from 16 to $96 . E\left(C_{D}\right)$ and $E(S t)$ represent the relative error on the drag coefficient and the relative error on the Strouhal number, respectively.

TAB. III shows $C_{D}$ and $S t$ for $R e=360$ as a function of grid size. For the coarsest grid (16 grid points per cylinder diameter) the error on $C_{D}$ is close to $40 \%$. Moreover, vortices are shed randomly and prevent from defining a characteristic vortex shedding frequency, and hence St. The error on $C_{D}$ is less than $20 \%$ for a grid size corresponding to 32 grid points per cylinder diameter. The wake (not shown here) consists of hairpin vortices which are not shed periodically. Indeed a second frequency manifests in the wake (close to one fourth of the expected frequency) and is a pure numerical artefact. It remains possible to define $S t$ based on the highest frequency, the resulting error is less than $5 \%$. The spurious frequency disappears when using a grid size corresponding to 64 grid points per cylinder diameter. For 
that case, TAB. III shows that the error on $C_{D}$ is about $5 \%$ while the error on $S t$ is less than 1\%. For the finest grid corresponding to 96 grid points per cylinder diameter, the error on $C_{D}$ is less than $3.5 \%$.

To summarize the findings of our mesh sensitivity analysis on cases representative of our physical analysis in the rest of this paper, it is necessary to distribute at least 3-4 grid points in the boundary layer around the cylinder (estimated as $D / R e^{1 / 2}$ ) to accurately describe the flow regimes we are interested in. When this condition is satisfied, our fictitious domain method yields accurate results for the flow past a yawed cylinder. Thereby, for the range of $R e \in[25,250]$ we intend to investigate in this paper, we use either 32 grid points per cylinder diameter for the low $25 \leq R e \leq 100$ cases, 48 grid points per cylinder diameter for the moderate $100<R e<150$ cases, and 64 grid points per cylinder diameter for the high $R e \geq 150$ cases. Corresponding meshes comprise between 10 to 100 millions of grid cells.

\section{FLOW PAST A YAWED $L / D=3$ CYLINDER}

We now investigate the flow past a $L / D=3$ cylinder for Reynolds numbers $R e \in[25,250]$ and yaw angles $\theta \in\left[0^{\circ}, 90^{\circ}\right]$. In total, more than eighty well resolved simulations are performed.

FIG. 3 illustrates the hydrodynamic regimes observed in a $(R e, \theta)$ plane. Colour areas delineate the regimes based on symmetry and unsteadiness. Three different temporal regimes are encountered: steady, periodic and unsteady. The regime is called periodic when the wake unsteadiness is predominantly governed by a unique frequency, otherwise it is called unsteady. Three types of symmetry can also be defined: (i) $x$ axial symmetry, (ii) $(x, z)$ horizontal reflectional planar symmetry, and (ii) $(x, y)$ vertical reflectional planar symmetry. We observe seven regimes classified by unsteadiness and symmetry properties in FiG. 3. For $\theta=0^{\circ}$, due to the specific geometric configuration, the wake is steady and axisymmetric for all $R e$ studied. It is likely that the axisymmetry will be broken for sufficiently higher $R e>250$ as observed for the $L / D=1$ cylinder (see Appendix). For $\theta>0^{\circ}$ the axisymmetry is intrinsically broken but the wake keeps a steady vertical symmetry plane. This regime (in yellow in FIG. 3) is the most frequently observed regime for the range of $R e$ studied. We also would like to shortly underscore the specific symmetry observed when the cylinder is perpendicular to the flow direction, i.e., $\theta=90^{\circ}$. Indeed for $R e<125$ and $\theta=90^{\circ}$ the 


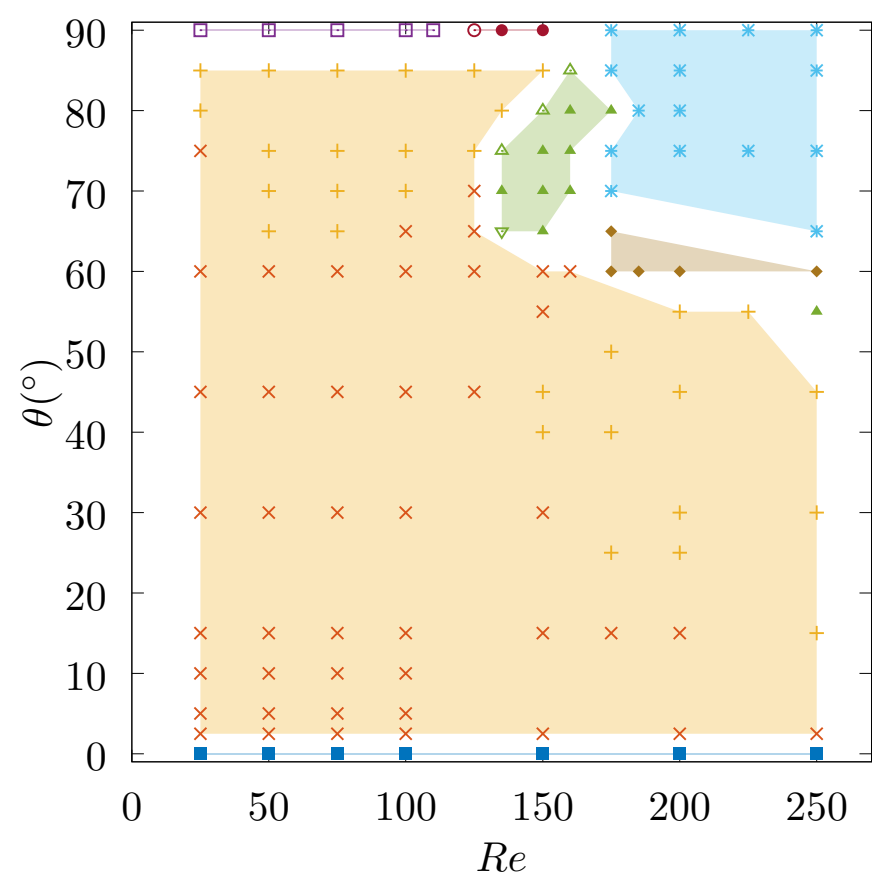

FIG. 3: Wake patterns past a $L / D=3$ cylinder as function of $\theta$ and Re. Coloured areas represent regimes by symmetry and unsteadiness. Blue : steady axisymmetric wake, yellow : steady symmetric wake with respect to the $(x, y)$ plane, purple : steady wake with two symmetry planes $((x, y)$ and $(x, z))$, green : periodic wake with one symmetry plane $(x, y)$, red : periodic wake with one symmetry plane $(x, z)$, brown : periodic shedding of quasi-symmetric double-sided hairpin vortices, cyan : 3D unsteady wakes. $\mathbf{0}$ : steady standing eddy or toroidal vortex, $\times$ steady shedding of one counter-rotating vortex pair, + steady shedding of 2 counter-rotating vortex pairs, $\square$ steady shedding of 2 symmetric counter-rotating vortex pairs, $\triangle$ periodic shedding of 2 counter-rotating vortex pairs $((x, y)$ symmetry plane $), \nabla$ periodic shedding of one counter-rotating vortex pair $((x, y)$ symmetry plane), $\odot$ periodic shedding of 2 counter-rotating vortex pairs $((x, z)$ symmetry plane), $\boldsymbol{\Delta}$ periodic shedding of single-sided hairpin shaped vortices, $\bullet$ periodic shedding of symmetric double-sided hairpin shaped vortices $((x, z)$ symmetry plane), * unsteady shedding of asymmetric double-sided hairpin shaped vortices, $\downarrow$ periodic shedding of quasi-symmetric double-sided hairpin vortices.

wake has two symmetry planes (purple region in FiG. 3). The onset of unsteadiness for all yaw angles is characterized by the emergence of a unique frequency, therefore those regimes are called periodic (green, red and brown regions in FIG. 3). Except for $\theta=60^{\circ}$, the onset 
of periodicity as a function of $R e$ is characterized by the appearance of one reflectional symmetry plane. The brown region in FIG. 3 is very specific in the sense that it is periodic but without a clear wake symmetry. It is called quasi-symmetric since each wake structure shed is almost parallel and its centroid follows a straight line. For the highest $R e$ and $\theta$, symmetry and periodicity are completely lost and three-dimensional structures manifest in the wake (cyan region in FIG. 3).

We also found three main wake patterns: standing eddy, longitudinal vortex pairs and hairpin shaped vortices. Hence to summarize, if we include wake pattern in the classification of regimes, we have found 10 regimes identified as symbols in Fig. 3. These various

hydrodynamic regimes and their associated transitions are hereafter detailed and discussed. In order to make the discussion as clear as possible, we discuss the flow regime map based on increasing $R e$ for decreasing ranges or specific values of $\theta$.

$$
\theta=90^{\circ}
$$

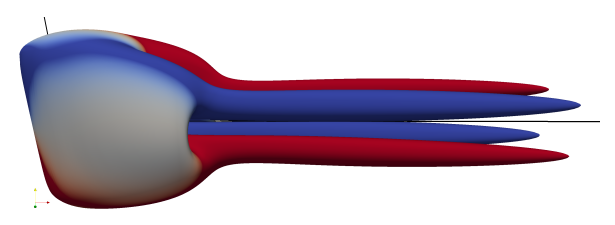

FIG. 4: Two steady symmetric counter-rotating vortex pairs at $\left(\theta=90^{\circ}, R e=100\right)$. $Q=0.01$ isosurfaces are coloured by the longitudinal vorticity ranging from -0.1 to 0.1 .

The dark lines starting at the centre of the cylinder indicate the cylinder axis and the streamwise direction.

Two steady symmetric counter-rotating vortex pairs Up to Re $\approx 120$ (FIG. 3, $\square$ ), the flow structure past the solid body oriented perpendicularly to the flow direction presents two steady symmetric counter-rotating vortex pairs displayed in FIG. 4. The regime is steady and symmetric with respect to the $(x, y)$ vertical plane and $(x, z)$ horizontal plane. The singular symmetric nature of the four vortices induces a zero-value of the lift force in both $y$ and $z$ directions. This wake pattern has been previously described by Inoue and Sakuragi $[25]$.

Periodic shedding of 2 counter-rotating vortex pairs From $R e \gtrsim 125$, the flow becomes unsteady and a periodic symmetric vortex shedding manisfests, as presented in FIG. 5. The 


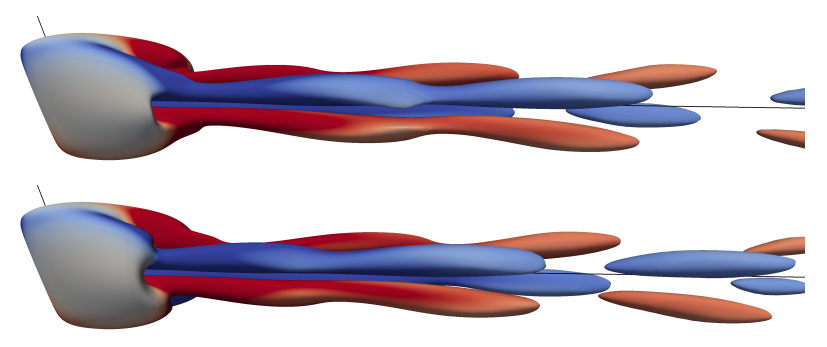

FIG. 5: Periodic shedding of two symmetric counter-rotating pairs at $\left(\theta=90^{\circ}, R e=125\right)$. The time interval between two frames is $\Delta t=5 . Q=0.004$ isosurfaces are coloured by the longitudinal vorticity ranging from -0.2 to 0.2 . The dark lines starting at the centre of the cylinder indicate the cylinder axis and the streamwise direction.

nature of the symmetry described above for $\left(\theta=90^{\circ}, R e<120\right)$ is altered and the $(x, y)$ vertical symmetry is broken by an unsteady mode while the horizontal symmetry plane is kept. FIg. 7a displays the force diagram for $R e=125$. The lift force along $z$ oscillates around a near-zero mean value $\left(\overline{C_{L z}}=1.136 \times 10^{-4}\right)$ with a low amplitude. The unsteady character of the solution has a weak impact on the drag force and the attractor looks like a vertical line. The lift force along $y$ is zero in agreement with the wake symmetry. The two pairs of vortices are wavy and oscillate with a dimensionless frequency $S t_{z}=0.109$ given by a Discrete Fourier Transform (DFT) analysis. The range of existence of this mode as a function of $R e$ is narrow (FIG. 3, $\odot$ ).

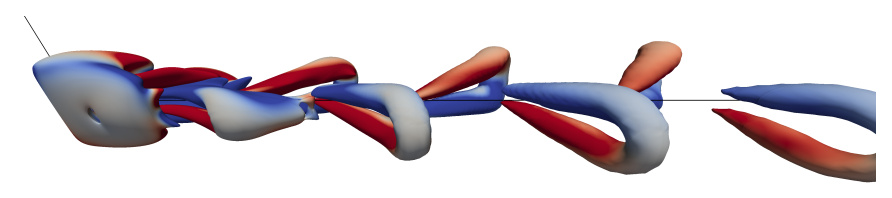

FIG. 6: Periodic shedding of double-sided horizontal hairpin vortices at $\left(\theta=90^{\circ}, R e=150\right) . Q=0.008$ isosurfaces are coloured by the longitudinal vorticity ranging from -0.2 to 0.2 . The dark lines starting at the centre of the cylinder indicate the cylinder axis and the streamwise direction.

Periodic shedding of symmetric double-sided hairpin shaped vortices A transition occurs for $R e \approx 135$. The wake pattern exhibits hairpin shaped vortex shedding (FIG. 3, •). Fig. 6 shows the flow structure obtained for $R e=150$. The hairpins are double-sided in the sense that alternated vortices of opposite rotational direction but of similar intensity are shed periodically. Our results agree well with the results of Inoue and Sakuragi [25]. In fact, 


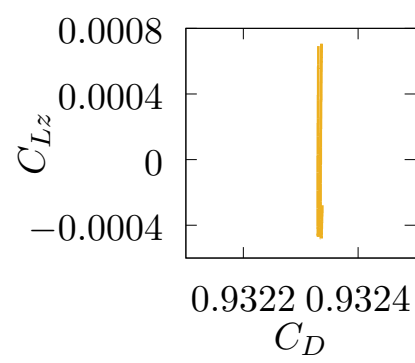

(a)

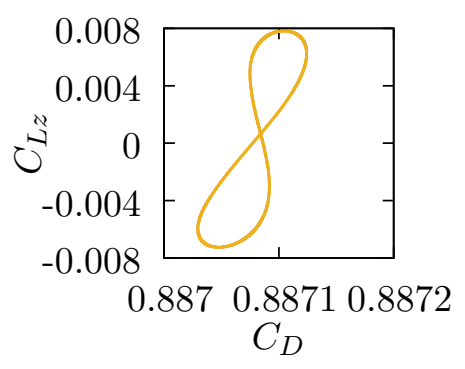

(b)

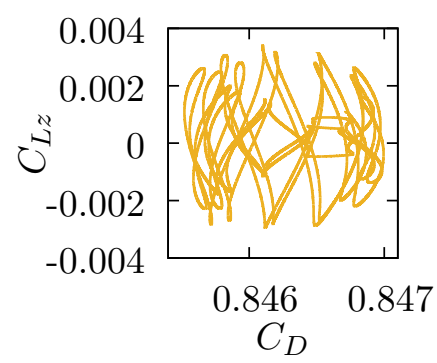

(c)

FIG. 7: $C_{D}-C_{L z}$ diagrams for the symmetric shedding of double-sided hairpin vortices at

$$
\left(\theta=90^{\circ}\right):(a) R e=125 \text {, (b) } R e=150 \text {, (c) } R e=175 .
$$

Inoue and Sakuragi [25] also observed two pairs of counter-rotating vortices for $R e=100$ and symmetric double-sided hairpin vortex shedding for $R e=150$.

The $C_{D}-C_{L z}$ diagram for $R e=150$ presented in FIG. 7b shows an attractor displaying an "eight" shape. The lift force mean value in the $z$ direction is $\overline{C_{L z}}=2.5 \cdot 10^{-4}$ and remains negligibly small. This implies, as observed on the wake pattern, that the hairpin vortices shed from both sides of the cylinder have the same intensity. The $C_{L z}$ oscillations amplitude is large in comparison to the $C_{D}$ oscillations amplitude. Moreover, the $C_{L z}$ oscillations amplitude is one order of magnitude larger than the $C_{L z}$ oscillations amplitude observed for $\left(\theta=90^{\circ}, R e=125\right)$. The emergence of hairpin vortices is related to an increase of the vortex intensity and is a distinguishable signature of the unsteady mode. The Strouhal number $S t_{z}=0.118$ is close to $S t_{z}$ found in the regime $\left(\theta=90^{\circ}, R e \approx 120\right)$. The $C_{D}$ and $C_{L z}$ oscillations are slightly out of phase since the extrema of $C_{D}$ occurs slightly shifted with respect to the extrema of $C_{L z}$. $C_{D}$ oscillates with a dimensionless frequency $S t_{x}=0.236$ equal to twice the leading dimensionless frequency in agreement with the symmetry observed for the wake pattern. The lift force in the $y$ direction is almost negligible in that case.

Unsteady shedding of asymmetric double-sided hairpin shaped vortices The Re $=175$ force diagram shown in Fig. 7c reveals the appearance of several frequencies in the wake. The leading dimensionless frequency along the transverse direction $S t_{z}=0.115$ is close to $S t_{z}$ found in the regime $\left(\theta=90^{\circ}, 135 \lesssim R e<175\right)$. A low frequency $S t_{z, \text { low }}=0.039$ of smaller amplitude also emerges. The drag force DFT shows a dominant dimensionless frequency $S t_{x}=0.272$ close to twice the leading dimensionless frequency in the transverse $z$ direction and a low dimensionless frequency $S t_{x, \text { low }}=0.0264$. The $y$ lift force amplitude, 
i.e., $C_{L y}$, is still weak (not shown here for the sake of conciseness) and of the order of one hundred times lower than $C_{L z}$ but is nonetheless increasing with $R e$. The regime observed for $R e=175$ can be seen as a transitional regime separating the shedding of symmetric hairpin vortices regime and the fully 3D wake regime.

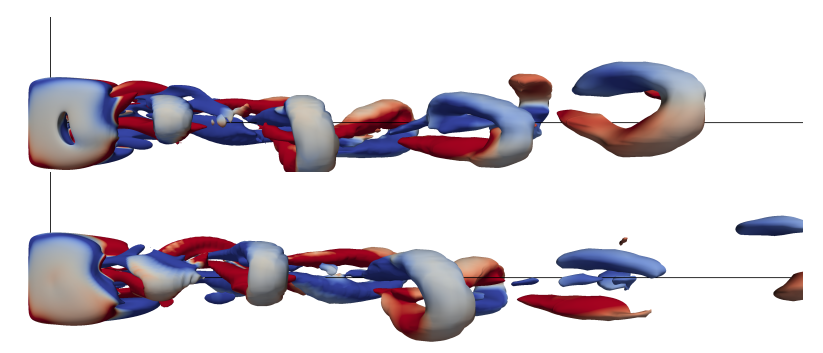

FIG. 8: 3D unsteady regime dominated by the shedding of horizontal double-sided hairpin vortices at $\left(\theta=90^{\circ}, R e=250\right)$. The time interval between two frames is $\Delta t=5$. $Q=0.008$ isosurfaces are coloured by the longitudinal vorticity ranging from -0.2 to 0.2 . The dark lines starting at the centre of the cylinder indicate the cylinder axis and the streamwise direction.

For $R e \gtrsim 200$, the force and torque diagrams reveal chaotic solutions and a clear loss of the $(x, z)$ reflectional planar symmetry. This wake is characterized by pseudo-chaotic flow structures (FIG. 3,*) and asymmetric double-sided hairpin vortices displayed in Fig. 8. Even if the solution becomes chaotic, the non-isotropic body geometry does not create a fully 3D behaviour of the flow in the near-wake. However the asymmetry is more pronounced as $R e$ increases. Finally the wake patterns for $\theta=90^{\circ}$ is similar to the wake pattern observed for lower $\theta$ in the range $\left[70^{\circ}, 90^{\circ}\right]$ as shown in the next section.

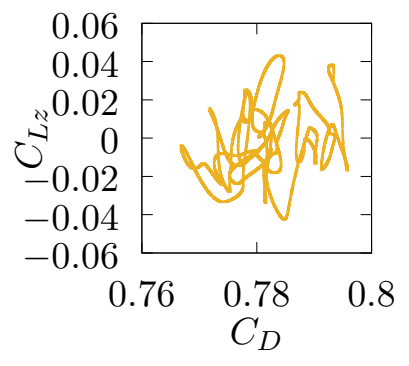

(a)

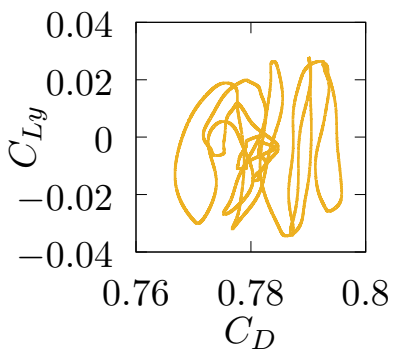

(b)

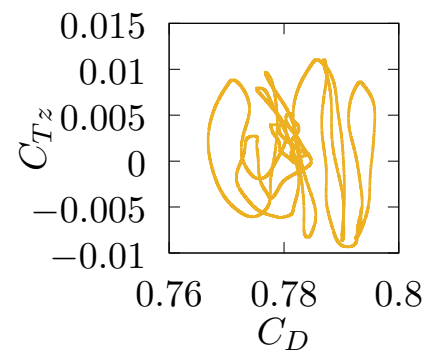

(c)

FIG. 9: Force and torque coefficient diagrams for the 3D unsteady regime dominated by the shedding of horizontal double-sided hairpin vortices at $\left(\theta=90^{\circ}, R e=250\right)$.

For $R e=250$. the $C_{D}$ and $C_{L z}$ oscillations amplitudes are at least one order of magnitude 
larger than the ones obtained for $\left(\theta=90^{\circ}, R e=175\right)$. The vortex shedding process is much more energetic. The $y$ lift force oscillation amplitude increases with $R e$ to reach a value close to the $z$ lift force oscillation amplitude, in other words $C_{L y}$ is getting closer to $C_{L z}$. A DFT analysis of the $z$ lift force for $R e=200$ gives a dominant dimensionless frequency $S t_{z}=0.115$ similar to the dimensionless frequency observed for lower Re. Consequently the leading frequency is weakly dependent on $R e$ in the range [125,200]. For $R e=250$, the more energetic unsteady mode has a dimensionless frequency $S t_{z}=0.152$, approximatively $32 \%$ higher than for all lower Re unsteady regimes investigated. However, due to the numerous other frequencies appearing in the wake, the definition of $S t$ is not straightforward. The $y$ lift force DFT gives $S t_{y, R e=200}=0.045$ and $S t_{y, R e=250}=0.046$. These pseudo-dominant dimensionless frequencies are at least twice smaller than the pseudo-dominant dimensionless frequency in the $z$ direction. For $R e \leq 175$, the associated torque magnitude in the $z$ direction remains weak (not shown here for the sake of conciseness) while for $R e \gtrsim 200$ the associated diagram presented in FIG. 9c shows a distinct and large torque. The torque oscillations amplitude, i.e., the $C_{T z}$ oscillations amplitude, increases with $R e$ and reaches a value close to the $C_{L y}$ oscillations amplitude at $R e=250$. These strong torque oscillations might explain the oscillations of the inclination angle observed experimentally for a $L / D=3$ cylinder falling under gravity for the same Reynolds number by Toupoint et al. [56].

$$
70^{\circ} \leq \theta<90^{\circ}
$$

Two pairs of steady counter-rotating vortices For $\theta \in\left[70^{\circ}, 90^{\circ}[\right.$ and for $\operatorname{Re} \in[25 ; 125]$ (FIG. 3, +), we observe two steady pairs of counter-rotating vortices past the yawed cylinder[Note2]. Unlike the $\theta=90^{\circ}$ cases, the horizontal symmetry is intrinsically broken by the body inclination. The bifurcation associated with this symmetry loss appears to be regular without hysteresis. The two pairs of vortices still have an opposite rotational direction but their intensity and shape differ as illustrated in Fig. 10a. The magnitude of the top vortex pair is lower than the magnitude of the bottom vortex pair. In this $\theta$ range, the difference of magnitude between the two pairs increases with decreasing $\theta$. The asymmetry is also identifiable in FIG. 10b. The streamlines obtained using a projected velocity field in a plane perpendicular to the streamwise direction located a few diameters downstream of the cylinder are shown. The rotational direction of each vortex is alternated. 


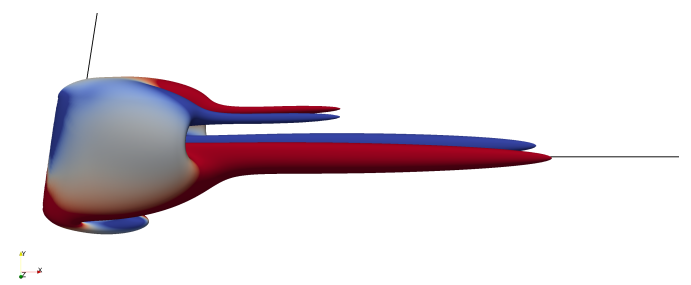

(a)

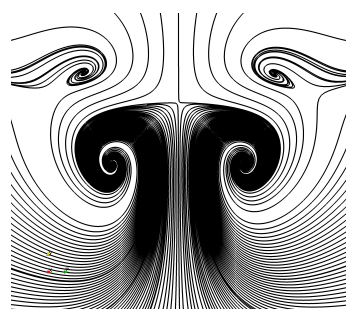

(b)

FIG. 10: Two pairs of asymmetric counter-rotating vortices at $\left(\theta=75^{\circ}, R e=100\right)$. (a) $Q=0.01$ isosurfaces are coloured by the longitudinal vorticity ranging from -0.2 to 0.2 .

The dark lines starting at the centre of the cylinder indicate the cylinder axis and the streamwise direction. (b) Streamlines in a $(y, z)$ plane perpendicular to the $x$ streamwise direction located at $5 D$ from the $x$ coordinate of the cylinder mass center.

The flow structure induces a non-zero value of the lift force in the $y$ direction.

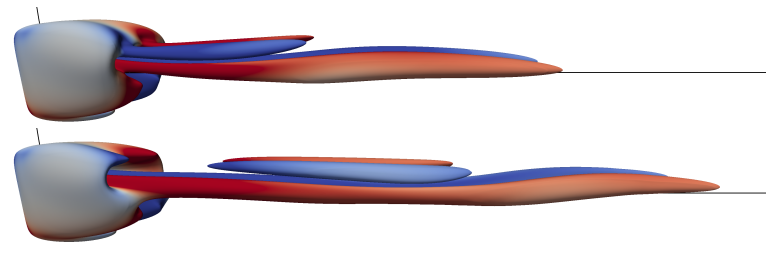

FIG. 11: Periodic shedding of two asymmetric counter-rotating vortex pairs at $\left(\theta=80^{\circ}, R e=150\right)$. The time interval between two frames is $\Delta t=10 . Q=0.004$ isosurfaces are coloured by the longitudinal vorticity ranging from -0.2 to 0.2 . The dark lines starting at the centre of the cylinder indicate the cylinder axis and the streamwise direction.

Periodic shedding of two counter-rotating vortex pairs For $R e>125$, a periodic solution appears. The onset of unsteadiness with respect to $R e$ is delayed by the horizontal symmetry loss. Indeed, while the first unsteady regime appears for $\theta \approx 90^{\circ}$ at $R e=125$, it only appears at $R e \approx 160$ for $\theta=85^{\circ}$. For smaller yaw angles $\theta<85^{\circ}$, the critical Reynolds number related to the appearance of unsteadiness decreases with decreasing $\theta$ (FIG. 3).

As shown in the two snapshots in FIG. 11 for $\left(\theta=80^{\circ}, R e=150\right)$, the wake structure oscillates. The top pair of vortices has a smaller magnitude than the bottom pair of vortices. During the whole sequence, one bottom pair of vortices and one top pair of vortices are shed. The intensity of the longitudinal vorticity oscillates and consequently the vortex filaments 
visually disappear depending on the $Q$ criterion value. This regime is here called periodic shedding of two counter-rotating vortex pairs (FIG. 3, $\triangle$ ).

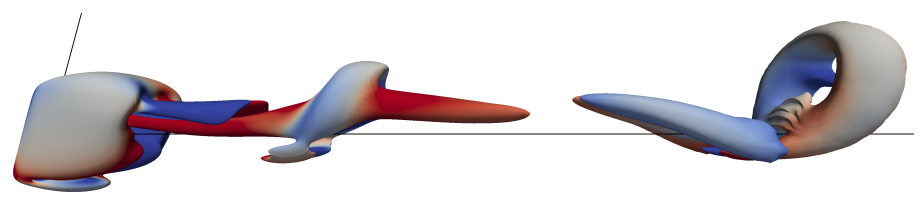

FIG. 12: Periodic shedding of single-sided hairpin vortices at $\left(\theta=75^{\circ}, R e=150\right)$. $Q=0.004$ isosurfaces are coloured by the longitudinal vorticity ranging from -0.2 to 0.2 .

The dark lines starting at the centre of the cylinder indicate the cylinder axis and the streamwise direction.

Periodic shedding of single-sided hairpins vortices Right after the steady-periodic transition, i.e., for slightly lower $\theta$ or slightly larger $R e$, we observe the shedding of single-sided hairpin vortices (FIG. 3, $\mathbf{\Delta}$ ). The hairpin vortices are shed in the $y$ direction and have the same orientation, as illustrated in FIG. 12 for $\left(\theta=75^{\circ}, R e=150\right)$. The vertical symmetry is preserved. This regime is called single-sided since, contrary to the double-sided regime, the hairpin vortices shed periodically have always the same rotational direction.

The first two unsteady regimes observed for this range of yaw angles ( $\triangle$ and $\boldsymbol{\Delta}$ in FIG. 3 ) are similar to the regime observed in the case of the flow past a sphere for $R e \in[210 ; 300]$ [52]. The periodic shedding of single-sided hairpin vortices is also observed for $L / D=1$ and $\left(\theta=0^{\circ}, R e=360\right)$, and described in Appendix .

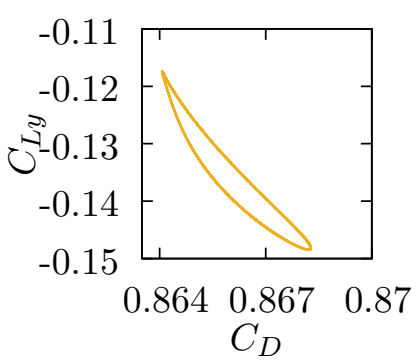

(a)

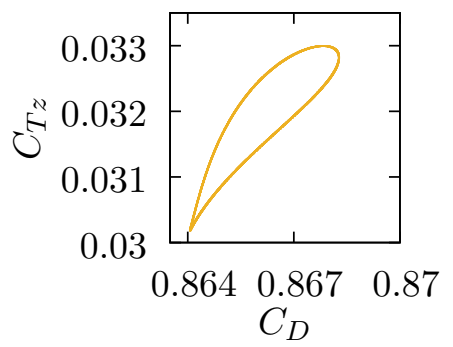

(b)

FIG. 13: Force and torque coefficient diagrams for the periodic shedding of single-sided hairpin vortices at $\left(\theta=75^{\circ}, R e=150\right)$ : (a) $C_{D}-C_{L y}$ and (b) $C_{D}-C_{T z}$.

The $C_{D}-C_{L y}$ diagram of this regime shown in FIG. 13a displays a single loop with a pointed extremity at the left top corner. This "pointed-like" behaviour increases with $R e$ 
(at $R e=135$, we observe a simple loop and no pointed extremity) and is observed for all the cases of shedding of single-sided hairpin vortices. The loop travels anticlockwise. The maximum of $C_{L y}$ happens slightly before the maximum of $C_{D}$ (not easy to see in FIG. 13a). The $C_{D}-C_{T z}$ diagram shown in FIG. 13b also reveals a single loop with a pointed extremety. The shift in time between the maximum of $C_{T z}$ and the maximum of $C_{D}$ is more visible on the top right corner of the loop. Force and torque are governed by a unique dimensionless frequency $S t_{y}=0.0478$. This frequency is close to the frequency encountered for $R e=135$ $\left(S t_{y, R e=135}=0.045\right)$.

Unsteady shedding of asymmetric double-sided hairpin vortices The value of Re corresponding to the transition between planar symmetry regimes and fully 3D regimes does not vary much when $70^{\circ} \leq \theta<90^{\circ}$. Indeed, the transition always occurs around $R e \approx 175$. However, the $3 \mathrm{D}$ wake pattern noticeably changes with $R e$ and $\theta$. This $3 \mathrm{D}$ regime region is characterized by the emergence of multiple frequencies in the wake. Two different cases are chosen to illustrate the different behaviours observed: $\left(\theta=85^{\circ}, R e=175\right)$ which is near the transition and $\left(\theta=75^{\circ}, R e=200\right)$ that exhibits a fully developed unsteady 3D wake. We define the shedding angle as the angle between the axis belonging to the symmetry plane of each vortex and passing through its extremity, and the horizontal axis passing through the body center.

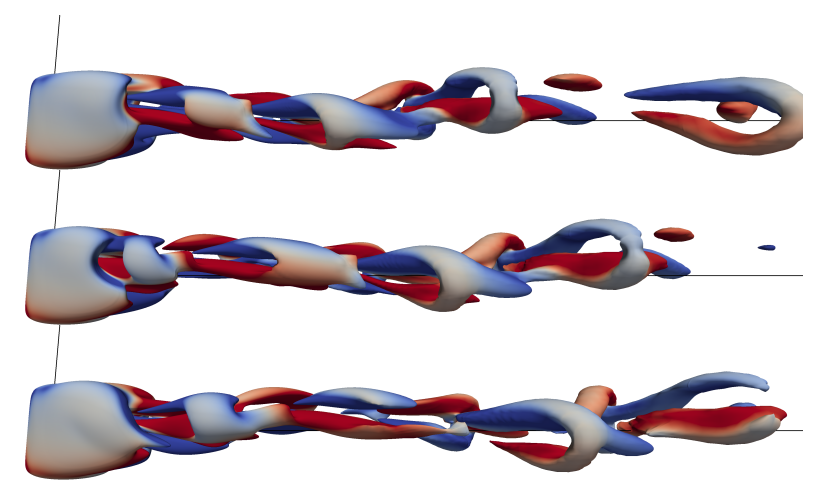

FIG. 14: Unsteady shedding of asymmetric double-sided hairpin vortices at $\left(\theta=85^{\circ}, R e=175\right)$. The time interval between two frames is $\Delta t=5 . Q=0.008$ isosurfaces are coloured by the longitudinal vorticity ranging from -0.2 to 0.2 . The dark lines starting at the centre of the cylinder indicate the cylinder axis and the streamwise direction.

Comparing FIG. 14 and FIG. 8 highlights that the shedding angle for $\left(\theta=85^{\circ}, R e=175\right)$ 
is smaller than the shedding angle for $\left(\theta=90^{\circ}, R e=250\right)$. Vortices are thus shed more "longitudinally" in the former case than in the latter case. FIG. 14 displays the location and orientation of the vortex oscillations in time in the $y$ direction. We observe that some hairpin vortices are slightly tilted with respect to others.

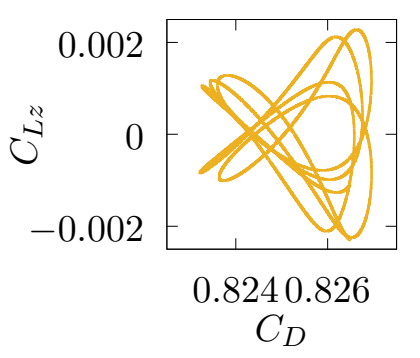

(a)

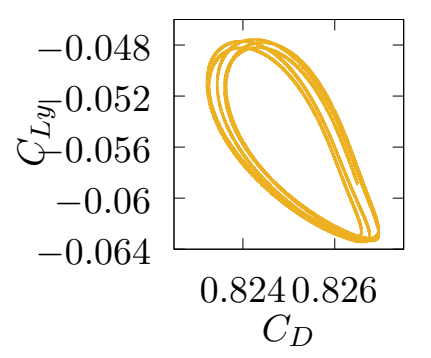

(b)

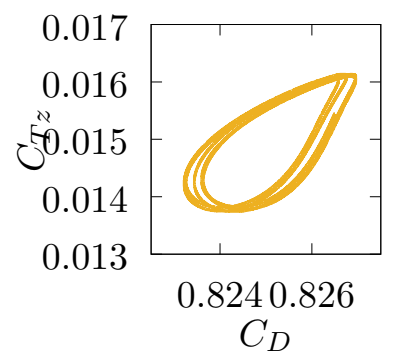

(c)

FIG. 15: Force and torque coefficient diagrams for the 3D unsteady regime dominated by the shedding of asymmetric double-sided hairpin vortices at $\left(\theta=85^{\circ}, R e=175\right)$.

According to the $C_{D}-C_{L z}$ force diagrams shown in FIG. 15a, the attractor looks like a butterfly. A DFT analysis of $C_{L z}$ gives a leading dimensionless frequency $S t_{z}=0.0708$ and a low dimensionless frequency $S t_{z, \text { low }}=0.022$ of smaller amplitude. The DFT analysis of $C_{D}$ gives $S t_{x}=0.0472$. These three non-harmonic frequencies explain the complicated but still identifiable modes of interaction in the $C_{D}-C_{L z}$ diagram. The $C_{D}-C_{L y}$ (respectively $C_{D}-$ $C_{T z}$ ) force diagram plotted in Fig. 15b (respectively c) shows a quasi-periodic attractor with a non-zero mean value of the lift force (respectively torque). The dimensionless frequency of the $y$ lift force oscillations $S t_{y}=0.0488$ for $\left(\theta=75^{\circ}, R e=175\right)$ is really close to $S t_{y}$ for $\left(\theta=75^{\circ}, R e=150\right)$. Moreover $C_{D}$ and $C_{L y}$ are almost in phase since the extrema of both occur at the same time. $C_{L z}$ is much smaller than $C_{L y}$, which indicates that the symmetry breaking and associated frequencies do not significantly impact the dynamics in the vertical direction. To conclude, the wake pattern and the force/torque diagrams for $\left(\theta=85^{\circ}, R e=175\right)$ indicate that this regime can be summarized as a superposition of two unsteady modes: double-sided hairpin shedding in the $z$ direction and an asymmetric pattern in the $y$ direction that follows from the shedding of single-sided hairpin vortices observed at lower Reynolds numbers.

Fig. 16 displays the wake structure for $\left(\theta=75^{\circ}, R e=200\right)$. In comparison to $(\theta=$ $\left.85^{\circ}, R e=175\right)$, vortices downstream of the body in the near wake and the far wake appear more interlaced and chaotic. The mean shedding angle (i.e., vortex inclination) is also more 


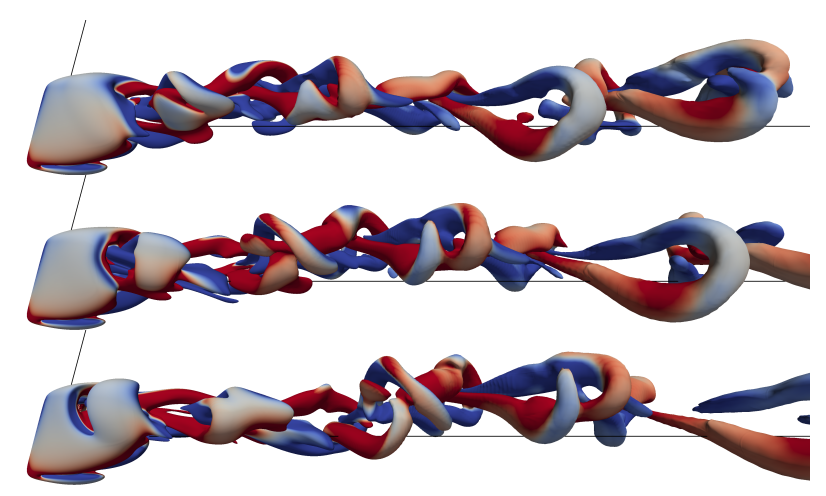

FIG. 16: Unsteady shedding of asymmetric double-sided hairpin vortices at $\left(\theta=85^{\circ}, R e=175\right)$. The time interval between two frames is $\Delta t=5 . Q=0.004$ isosurfaces are coloured by the longitudinal vorticity ranging from -0.2 to 0.2 . The dark lines starting at the centre of the cylinder indicate the cylinder axis and the streamwise direction.

pronounced.

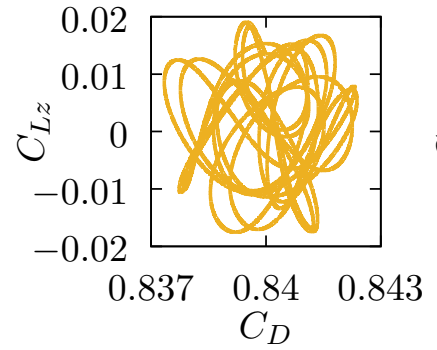

(a)

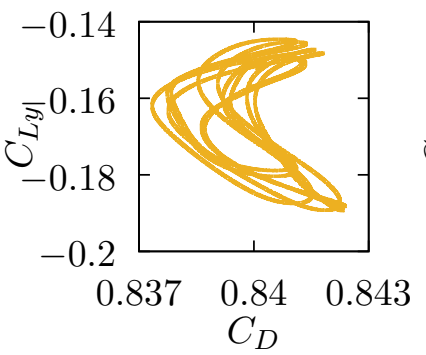

(b)

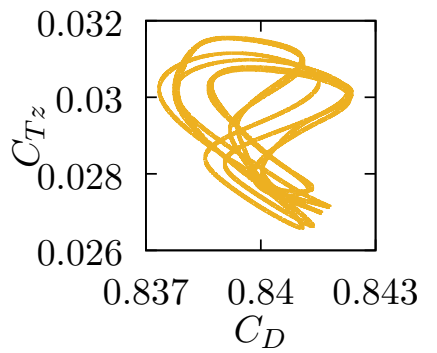

(c)

FIG. 17: Force and torque coefficient diagrams for the 3D unsteady regime dominated by the shedding of asymmetric double-sided hairpin vortices at $\left(\theta=75^{\circ}, R e=200\right)$.

The chaotic nature of the flow is markedly visible in the force/torque diagrams presented in Fig. 17a-c. The $C_{D}-C_{L z}$ diagram plotted in FIG. 17a shows a kind of wool ball shape while the $C_{D}-C_{L y}$ and $C_{D}-C_{T z}$ diagrams show a more distinct signal that looks like a disturbed height shape. These shapes differ significantly from the shapes observed for $\left(\theta=85^{\circ}, R e=175\right)$. The magnitude of the $C_{L z}$ and $C_{L y}$ coefficients are about one order of magnitude larger than for $\left(\theta=85^{\circ}, R e=175\right)$. The $C_{L z}$ oscillations amplitude is similar to the $C_{L y}$ oscillations amplitude. Therefore the $(x, y)$ symmetry plane is distinctly broken. The mean value of $C_{L z}$ approaches zero, i.e., $\overline{C_{L z}} \simeq 0$. The dimensionless frequency of the $y$ and 
$z$ lift force oscillations are $S t_{y}=0.0516$ and $S t_{z}=0.1288$, respectively. At least two vortices are shed during one oscillation period in the $y$ direction. $S t t_{y}$ for $\left(\theta=85^{\circ}, R e=200\right)$ is close to $S t_{y}$ for $\left(\theta=85^{\circ}, R e \leq 175\right)$. As for $\left(\theta=85^{\circ}, R e=175\right)$, and despite its apparent chaotic nature, the case $(\theta=75, R e=200)$ can be seen as the superposition of two unsteady modes : one along $y$ and the other along $z$. The chaotic interaction between these perpendicular modes is representative of the cyan coloured region in FIG. 3 called 3D unsteady regime.
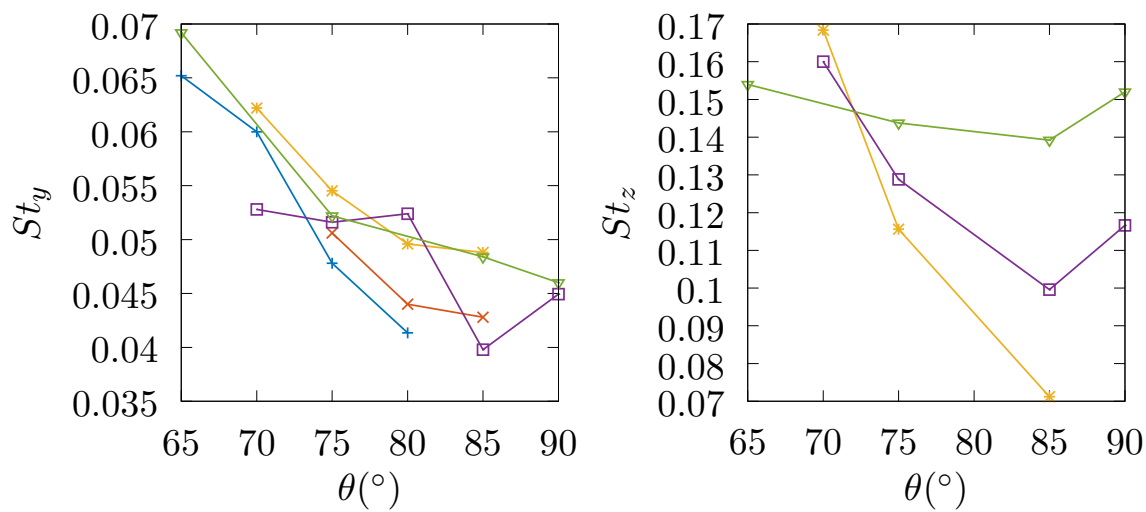

FIG. 18: Strouhal number $S t$ as a function of the yaw angle $\theta$ at $R e=150(+), R e=160$ $(\times), R e=175(*), R e=200(\square)$ and $R e=225$ ( $\nabla$ : (left) oscillations in the $y$ vertical direction, (right) oscillations in the $z$ horizontal direction.

Evolution of the Strouhal number Figs. 18a and b present the Strouhal numbers for $65^{\circ} \leq \theta \leq 90^{\circ}$ and $150 \leq R e \leq 250$ [Note3], respectively. In these ranges 4 unsteady regimes occur: periodic shedding of one or two pairs of counter-rotating vortices, periodic shedding of single-sided hairpin vortices and unsteady asymmetric shedding of double-sided vortices. For all Re studied, $S t_{y}$ exhibits a decreasing trend when $\theta$ increases as shown in Fig. 18a. A slight increase of $S t_{y}$ with $R e$ for all $\theta \in\left[65^{\circ}, 90^{\circ}\right]$ is also observed for $150 \leq R e \leq 175$. St $t_{z}$ decreases with the increase of $\theta$ for $\theta \in\left[65^{\circ}, 85^{\circ}\right]$ and slightly increases with the increase of $\theta$ for $\theta \in\left[85^{\circ}, 90^{\circ}\right]$, as shown in FIG. 18b. This interesting trend is contradictory to what the IP predicts, where $S t_{z}$ is supposed to behave as $\sin \theta$ and hence to constantly increase [47]. The possible reason explaining this discrepancy is that $L / D=3$ is not sufficiently large to apply the IP theory. For $\theta \in\left[75^{\circ}, 90^{\circ}\right]$ and $R e \in[175,225], S t_{z}$ increases strongly with $R e$.

Comments on the $\theta=70^{\circ}$ case So far, we have not discussed the case of the yaw angle $\theta=70^{\circ}$. Indeed this yaw angle and its associated regimes are indeed at the transition between regimes observed for $70^{\circ} \leq \theta<90^{\circ}$ and $60^{\circ} \leq \theta<70^{\circ}$. At this stage, we can say the 
following. For $\theta \simeq 70^{\circ}$, the single-sided hairpin vortex shedding regime is the first unsteady regime encountered after the steady pair of counter-rotating vortices regime (detailed in the next section). A more refined analysis in the range $R e \in[125 ; 135]$ might show an intermediate regime corresponding to the periodic shedding of one pair of counter-rotating vortices (also detailed in the next section). Nevertheless, we have decided to classify this specific yaw angle $\theta=70^{\circ}$ in the $70^{\circ} \leq \theta<90^{\circ}$ category as most transitions observed for $70^{\circ} \leq \theta<90^{\circ}$ do also occur for $\theta \simeq 70^{\circ}$ and in particular the transition between single-sided hairpin vortices and unsteady shedding of double-sided hairpin vortices.

$60^{\circ} \leq \theta<70^{\circ}$

Steady shedding of a pair of counter-rotating vortices For $60^{\circ} \leq \theta<70^{\circ}$ and $R e \lesssim 125$, the steady state solution with a vertical symmetry plane changes markedly as illustrated in FIG. 3. Indeed for $\theta>65^{\circ}$ we preferentially found double pairs of counter-rotating vortices while for $\theta \approx 60^{\circ}$ we found a single pair of counter-rotating vortices. No hysteresis is found between these two regimes: the extra vortex pair, visualized with the $Q$ criterion, slowly vanishes as $\theta$ decreases. No symmetry-breaking and no loss of steadiness are associated with this transition. Therefore this transition is not a real bifurcation (or an interaction with an additional unstable mode) but only a continuous and smooth evolution of the wake pattern.

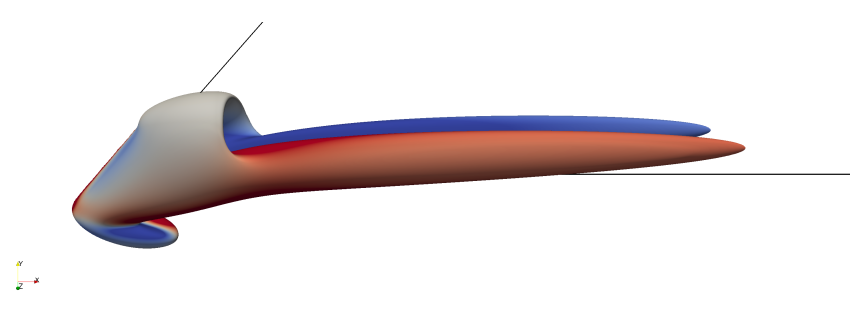

(a)

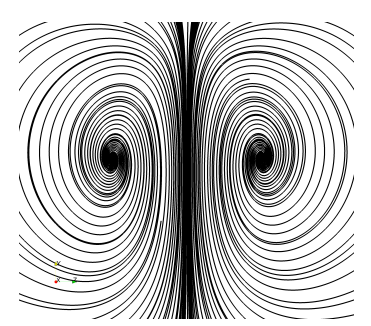

(b)

FIG. 19: One steady counter-rotating vortex pair at $\left(\theta=45^{\circ}, R e=75\right)$ : (a) $Q=0.004$ isosurfaces are coloured by the longitudinal vorticity ranging from -0.2 to 0.2 . The dark lines starting at the centre of the cylinder indicate the cylinder axis and the streamwise direction. (b) Streamlines in a $(y, z)$ plane perpendicular to the $x$ streamwise direction located at $5 D$ from the $x$ coordinate of the cylinder mass center.

The single pair of counter-rotating vortices regime is also the regime that prevails for 
$R e \lesssim 125$ and lower yaw angles $0^{\circ}<\theta \leq 60^{\circ}$. In fact, FIG. 19a shows a pair of counterrotating vortices in the wake of the cylinder for $\left(\theta=45^{\circ}, R e=75\right)$ that looks like the arms of a squid. This regime has been observed for the flow past a sphere by Johnson and Patel [28], past $L / D \geq 20$ long cylinders with flat ends by Ramberg [47] and past long pointed-end cylinders at high Reynolds numbers and small yaw angles by [17]. This pattern is also called bifid wake by [18] in the case of the flow past a sphere. In Fig. 19a, the region below the cylinder also presents a distinctive bulge of opposite vorticity contours. The entire wake keeps a $(x, y)$ reflectional planar symmetry. Fig. 19b shows the streamlines of the projected velocity in a plane perpendicular to the streamwise direction located a few diameters downstream of the cylinder for $\left(\theta=45^{\circ}, R e=75\right)$. We can clearly see two opposite longitudinal vortices.

Onset of unsteadiness The critical Reynolds number for onset of unsteadiness increases markedly when decreasing $\theta$ for $60^{\circ} \leq \theta<70^{\circ}$ as depicted in FIG. 3. For $R e \in[125 ; 185]$, depending on $\theta$, we observe two different transitions to unsteadiness. For $\theta=65^{\circ}$, we observe a transition from steady shedding of one counter-rotating vortex pair to periodic shedding of one counter-rotating vortex pair while for $\theta=60^{\circ}$ we observe a transition from steady shedding of one counter-rotating vortex pair to quasi-symmetric shedding of double-sided vortices. Moreover, the path to the 3D unsteady regime when increasing $R e$ is particularly complex for $\theta=65^{\circ}$. With increasing $R e$, we found the following succession of regimes: periodic shedding of one pair of counter-rotating vortices, periodic shedding of single-sided hairpin vortices and eventually quasi-symmetric shedding of double-sided vortices. Reasoning in term of bifurcations, this region $R e \in[125 ; 185]$ is a battlefield of at least one steady mode and two unsteady modes. These multiple interactions and associated bifurcations are currently unclear and we would like to point out that this $(R e, \theta)$ region may reveal other solution types. In the following the new regimes observed in that region are described and a very brief discussion is provided on the transition scenario.

Periodic shedding of a pair of counter-rotating vortices This regime occurs for $(\theta=$ $\left.65^{\circ} ; R e=135\right)$ and is characterized by a vertical symmetry plane. FIG. 20 illustrates the wake pattern observed in that case. In comparison to the steady shedding of one counterrotating vortex pair, the pair of vortices here twists with respect to their center line. The dimensionless vortex shedding frequency is $S t_{y}=0.067$

When Re increases, periodic shedding of a pair of counter-rotating vortices transitions 


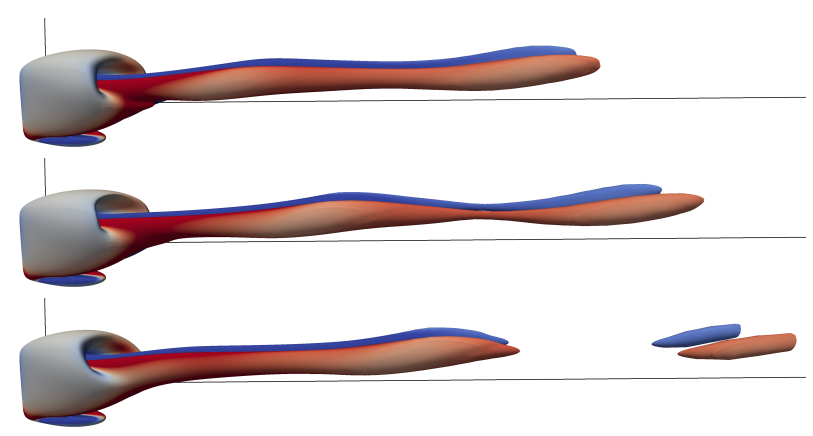

FIG. 20: Periodic shedding of one counter-rotating vortex pair at $\left(\theta=65^{\circ}, R e=135\right)$. The time interval between two frames is $\Delta t=5 . Q=0.004$ isosurfaces are coloured by the longitudinal vorticity ranging from -0.2 to 0.2 . The dark lines starting at the centre of the cylinder indicate the cylinder axis and the streamwise direction.

to shedding of single-sided hairpin vortices. This shedding of single-sided hairpin vortices regime and the associated transition are similar to what we observed for $70^{\circ} \leq \theta<90^{\circ}$ and have been described in details in Section. We observe another bifurcation in the range $150 \leq R e \leq 175$ that leads to quasi-symmetric shedding of double-sided hairpin vortices. This regime takes place after a very long transient regime characterized by the shedding of single-sided hairpin vortices. This transition is quite unusual in the present context, since the wake (steady for $\theta=60^{\circ}$ or periodic for $\theta=65^{\circ}$ ) looses entirely its vertical planar symmetry to reach a quasi-symmetric regime characterized by a zero $y$ lift force. This unsteady regime prevails for $60^{\circ} \leq \theta<65^{\circ}$ and $R e>175$.

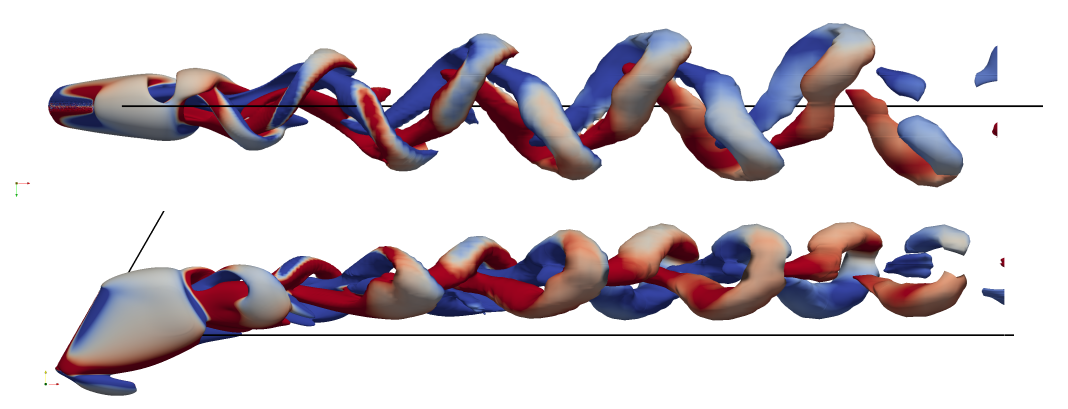

FIG. 21: The "accordion fold" regime or periodic shedding of quasi-symmetric double-sided hairpin vortices at $\left(\theta=60^{\circ}, R e=200\right)$. $Q=0.008$ isosurfaces are coloured by the longitudinal vorticity ranging from -0.2 to 0.2 . The dark lines starting at the centre of the cylinder indicate the cylinder axis and the streamwise direction. 
Periodic shedding of quasi-symmetric double-sided hairpin vortices FIG. 21 shows two perpendicular views of the typical wake pattern observed for $60^{\circ} \leq \theta<65^{\circ}$ and $R e>175$, i.e., in the brown region in FIG. 3. The 3D vortex structures are well organized even if no symmetry plane can be rigorously defined. The hairpins shed from both sides of the cylinder are parallel. Moreover the hairpin cores are alternatively transported on two parallel longitudinal and rectilinear paths. This regime is thus called periodic shedding of quasisymmetric double-sided hairpin vortices.

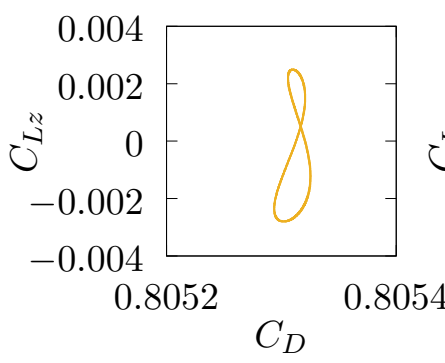

(a)

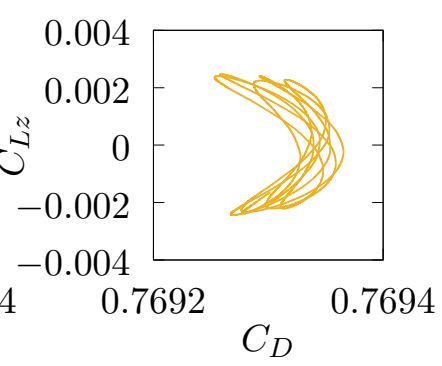

(b)

FIG. 22: $C_{D}-C_{L z}$ diagrams for the periodic shedding of quasi-symmetric double-sided hairpin vortices at $\theta=60^{\circ}$ : (a) $R e=200$, (b) $R e=250$.

FIG. 22 shows the force diagrams for $\theta=60^{\circ}$ and two different Reynolds numbers $R e=$ 200 and $R e=250$. The attractor for $R e=200$ shows a " 8 " shape similar to the shape observed for the $\left(\theta=90^{\circ}, R e=150\right)$ shedding of double-sided vortices regime. The visible similarity with the shedding of symmetric double-sided hairpin vortices regime justifies its "quasi-symmetric" name. The $C_{D}$ oscillations amplitude is much smaller than the $C_{L z}$ oscillations amplitude. Figs. 22a and b show that for both Re the amplitude of oscillation of the $z$ lift force is similar. In this regime and for both $R e$, the lift force in the $y$ direction (not shown here) has a steady non-zero value. Therefore this regime can be seen as the superposition of two perpendicular modes : one is a steady mode with a non-zero lift along $y$ and an unsteady small amplitude mode oriented along $z$.

In the region of periodic shedding of quasi-symmetric double-sided hairpin vortices, the Strouhal number increases with the Reynolds number as shown in Fig. 23. The trend followed by the computed $S t_{z}$ is opposite to the trend predicted by the IP. For all $R e$, the computed $S t_{z}$ decreases slightly when increasing $\theta$. Moreover, $S t_{z}$ computed in this regime is close to $S t_{z}$ computed in other flow regimes where double-sided hairpin vortices 


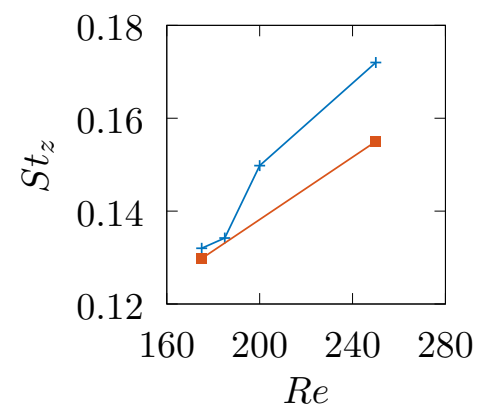

FIG. 23: $S t_{z}$ as a function of $R e$ for $\theta=60^{\circ}(+)$ and $\theta=65^{\circ}(\mathbf{a})$.

are observed.

Finally, the wake pattern is quite unusual and is (presumably improperly) renamed the 'accordion fold' regime due to the specific position of the hairpin vortices. In the flow map shown in FIG. 3, the 'accordion fold' regime is surrounded by 3D unsteady and single-sided regimes. This raises questions about how unstable modes grow and compete with each other. A small change of the cylinder tilt angle $\theta$ by a few degrees drastically changes the symmetry/lack of symmetry and associated wake patterns. This question could be further elucidated by the bifurcation theory $[12,16]$.

Unsteady shedding of double-sided hairpin vortices Keeping $\theta=65^{\circ}$ fixed and increasing $R e$ up to $R e=250$ leads to a transition from periodic shedding of quasi-symmetric doublesided hairpin vortices to unsteady shedding of asymmetric double-sided hairpin vortices. This regime is not shown here as it has been shown and discussed for larger $\theta$ (see cyan region in Fig. 3 and Fig. 16 for the wake pattern). It is characterized by $C_{L y}$ oscillations of same amplitude as that of $C_{L z}$ oscillations. The Strouhal number of the leading mode in the $y$ direction and $z$ direction is $S t_{y}=0.07$ and $S t_{z}=0.155$, respectively. Hence, $S t_{z}$ is about twice larger than $S t_{y}$. As emphasized before, the unsteady shedding of asymmetric double-sided hairpin vortices regime can be basically seen as the superposition of at least one mode along $z$, which in this $\theta$ range appears for lower $R e$, and one mode along $y$.

$$
\theta=55^{\circ}
$$

For $\theta=55^{\circ}$ the onset of unsteadiness is characterized by the transition from steady shedding of two vortex pairs to shedding of single-sided hairpin vortices. We estimate that the transition occurs around $R e \approx 230$ as the case $R e=225$ is still steady but very close to 
become unsteady. The transition between these two regimes has been described before and we only make short additional comments below.

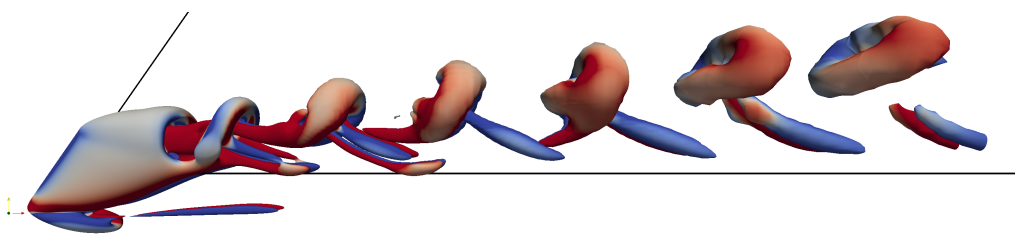

FIG. 24: Original periodic shedding of single-sided hairpin vortices at $\left(\theta=55^{\circ}, R e=250\right)$. $Q=0.008$ isosurfaces are coloured by the longitudinal vorticity ranging from -0.2 to 0.2 .

The dark lines starting at the centre of the cylinder indicate the cylinder axis and the streamwise direction.

Original periodic shedding of single-sided hairpin vortices FIG. 24 displays the wake pattern for $\left(\theta=55^{\circ}, R e=250\right)$ and exhibits single-sided hairpin vortices with vertical planar symmetry. However some differences are noticeable in comparison with the shedding of single-sided hairpin vortices observed for $70^{\circ} \leq \theta \leq 85^{\circ}$. The size of vortices is visibly smaller in Fig. 24 than in FIG. 13. Hairpin vortices are shed not only along $x$ but also along $y$, since the vortices monotonously move away from the $x$ axis in the cylinder wake. We also observe a pair of counter-rotating vortices at the bottom of the cylinder that was not present for $70^{\circ} \leq \theta \leq 85^{\circ}$. Finally the dimensionless vortex shedding frequency is $S t_{y}=0.1716$. This value is as least twice larger than the largest dimensionless frequency obtained in the range $70^{\circ} \leq \theta \leq 85^{\circ}$ and relates to the larger number of vortices observed in FIG. 24 compared to FIG. 13. The $C_{D}-C_{L z}$ diagram shows a simple closed loop of very small amplitude (not shown here) as observed in the range $70^{\circ} \leq \theta \leq 85^{\circ}$.

$$
0^{\circ}<\theta<55^{\circ}
$$

The steady shedding of one or two pairs of counter-rotating vortices are the two wake patterns observed for $\theta=0^{\circ}$ up to $\theta \approx 55^{\circ}$ for all Re. These regimes have been documented in Section and Section. No periodic regime is observed and the vertical planar symmetry is always present. As shown in FIG. 3, the one pair regime prevails for $R e \leq 150$ while the two pair regime increasingly dominates for higher $R e$ and increasing $\theta$. 


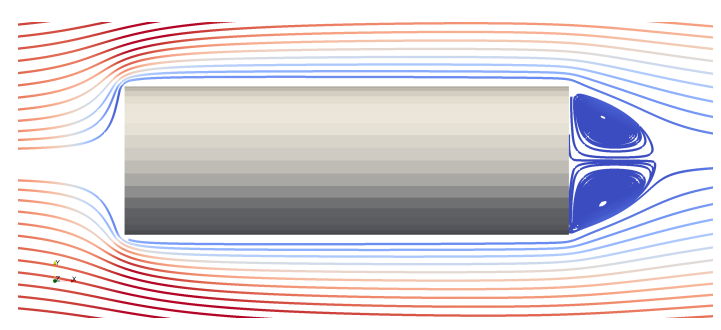

FIG. 25: Steady toroidal vortex downstream of a $\theta=0^{\circ}$ cylinder at $R e=50$. Streamlines pattern $(x, y)$ plane perpendicular to the $z$ transverse containing the cylinder axis are coloured by the dimensionless axial velocity (dark blue $u_{x}=0$, red $u_{x}=1$ ).

$\theta=0^{\circ}$

Standing toroidal eddy The aligned cylinder case completes the flow map shown in FIG. 3. Due to the specific symmetry of this configuration, the wake stays axisymmetric for all considered $R e$. The wake is characterized by a steady recirculating eddy as illustrated in FIG. 25 for $\left(\theta=0^{\circ}, R e=50\right)$. This is a distinguishable feature of the flow past a bluff body at Reynolds numbers higher than 10 [5, p. 259]. When the body is angular with salient edges, the detachment of the boundary layer usually occurs at the edge of the body [5, p. $329]$.

The length of the recirculation region $l_{r}$ increases with $R e$ as plotted in FiG. 26. We also plot in FIG. 26 the recirculation length as a function of $R e$ for an aspect ratio $L / D=1$. For both aspect ratios, $l_{r}$ increases as $\sqrt{R e}$. In the case of the flow past a sphere, $l_{r}$ versus Re follows a logarithmic law [55], while a linear law has been found in the case of the flow past an infinite cylinder [10]. The mathematical form of the relationship between $l_{r}$ and $R e$ thus depends on the geometry of the obstacle.

The transition from the steady recirculating eddy regime to a periodic or unsteady vortex shedding regime for the flow past a $L / D=3$ cylinder is expected to occur for higher Reynolds number as observed for the flow past a $L / D=1$ cylinder (see Appendix ).

\section{Additional comments on aspect ratio}

The flow map shown in Fig. 3 and the analysis of the flow regimes that we have performed so far reveal the strong dependence of the wake pattern on both $R e$ and $\theta$. We would like to shortly comment here on the transition between some regimes in light of results available in 


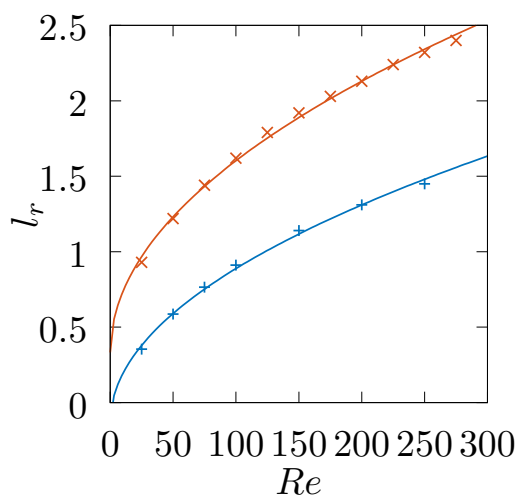

FIG. 26: Recirculation length $l_{r}$ of an aligned cylinder with the flow direction with respect to the Reynolds number. $+: L / D=3$ cylinder. $\times: L / D=1$ cylinder (Appendix). The solid lines correspond to fits of our numerical results of the $a R e^{1 / 2}+b$ form, where $a$ and $b$ are the fitting parameters.

the literature. The results of Ramberg [47] for long cylinders $(L / D \geqslant 20,160 \leq R e \leq 1000)$ (FIG. 2c in his article) are valuable data to discuss similarities and differences with our own results presented in this work. Ramberg [47] observed two different regimes: (i) a steady regime with one counter-rotating vortex pair for low $\theta$ and (ii) a periodic shedding of oblique vortices for high $\theta$ (Von-Karman vortex street). The transition between these two regimes occurs approximatively at $\theta=40^{\circ}$ and decreases with $R e$. There are two main differences between the map drawn by Ramberg [47] and ours. Firstly, he observed oblique vortex shedding for high $\theta$, while we observe hairpin vortex shedding. Secondly, the transition between the regimes observed by Ramberg [47] and the transition between our regimes does not occur for the same Re and $\theta$. These differences are attributed to different aspect ratios in Ramberg [47]'s study and ours. Inoue and Sakuragi [25], when studying the flow past a cylinder in a cross flow, also pointed out that for a fixed Reynolds number the aspect ratio has a significant impact on the wake pattern. For instance, at $R e=150$, hairpin vortex shedding is observed for a $L / D=3$ cylinder while oblique vortex shedding is observed for a $L / D=25$ cylinder.

\section{DRAG, LIFT AND TORQUE COEFFICIENTS}

In this section we suggest simple laws for the drag force, the lift force and the hydrodynamic torque as a function of $\theta$ and $R e$. We first shortly review different approaches to this 
problem from the literature and then suggest simple laws for $C_{D}, C_{L y}$ and $C_{T z}$. We then put the suggested laws to the test by comparing their performance to our computed results.

\section{State of the art}
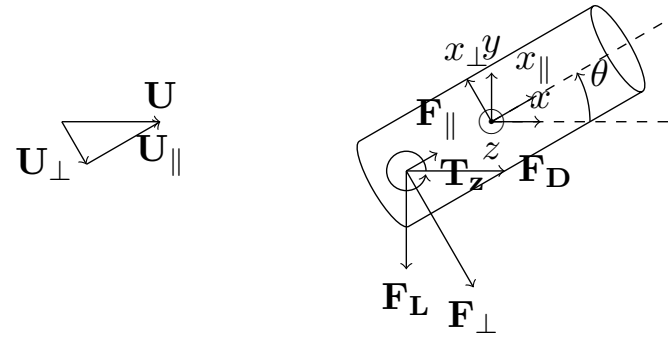

FIG. 27: Sketch of the force and torque exerted on a yawed cylinder.

The force and torque experienced by a finite-length cylinder in a steady flow are not known exactly even in the Stokes flow regime $R e=0$. However, owing to the linearity of the equations in this regime, the force exerted on a cylinder tilted with an angle $\theta$ (see FiG. 27) can be related to the force exerted on the same cylinder tilted by $\theta=0^{\circ}$ and $\theta=90^{\circ}$ as:

$$
\begin{gathered}
C_{\perp}=C_{\perp \theta=90^{\circ} \sin \theta} \\
C_{\|}=C_{\| \theta=0^{\circ}} \cos \theta
\end{gathered}
$$

where $C_{\perp}$ and $C_{\|}$are the normal force and parallel force coefficients, respectively. The drag and lift coefficients follow as:

$$
\begin{aligned}
C_{D} & =C_{D \theta=0^{\circ}} \cos ^{2} \theta+C_{D \theta=90^{\circ}} \sin ^{2} \theta \\
C_{L y} & =C_{D \theta=0^{\circ}} \cos \theta \sin \theta-C_{D \theta=90^{\circ}} \sin \theta \cos \theta
\end{aligned}
$$

In the Stokes regime, the hydrodynamic torque on a cylinder is zero : a freely moving cylinder keeps its initial orientation while falling under gravity. This specific property is lost when including weak effects of inertia [11]. When $R e \ll 1$, the torque coefficient along $z$ varies as :

$$
C_{T z} \propto \sin \theta \cos \theta
$$

For high Reynolds numbers, the IP states that the normal force on an infinitely long yawed cylinder in a flow of velocity $U$ is the same as the force exerted on the same cylinder 
placed in a cross flow of velocity $U \sin \theta$ [54]. The normal coefficient can thus be written as [20]:

$$
C_{\perp}=C_{\perp \theta=90^{\circ}} \sin ^{2} \theta
$$

and the drag and lift coefficients as:

$$
\begin{aligned}
C_{D} & =C_{D \theta=90^{\circ}} \sin ^{3} \theta \\
C_{L y} & =C_{D \theta=90^{\circ}} \sin ^{2} \theta \cos \theta
\end{aligned}
$$

In order to account for the force experienced by a cylinder aligned with the flow direction into the drag law, Rosendahl [50] proposed the following empirical relation based on the IP:

$$
C_{D}=C_{D \theta=0^{\circ}}+\left(C_{D \theta=90^{\circ}}-C_{D \theta=0^{\circ}}\right) \sin ^{3} \theta
$$

There are several other empirical and semi-empirical laws derived for the drag force exerted on non spherical-particles as, e.g., the law suggested by [21]. In [21], the authors proposed to use the lengthwise and crosswise sphericity (whose definition can be found in their article) instead of using explicitly the orientation of the particle. Their correlation gives good agreement with existing results in the literature when dealing with general complex shape particles. Here we are interested in cylindrical particles and attempt to develop a specific law for such a body shape.

\section{Simple laws for the hydrodynamic force and torque}

One of the drawbacks of the laws proposed above is that, for most of them, they explicitly depend on the drag force exerted on the cylinder when it is aligned with the flow direction, i.e., $C_{D \theta=0^{\circ}}$. The flow past a cylinder perpendicular to the flow direction has been studied extensively and a precise correlation has been proposed by Clift et al. [9, p. 154]. This is not the case when the cylinder is aligned with the flow direction. Therefore for practical purposes it is desirable to find a law that does not involve $C_{D \theta=0^{\circ}}$ explicitly. Moreover, the validity of the laws proposed in the previous subsection as function of Re has not been assessed for the present range of $R e \in[25,250]$. Our intention here is to provide simple laws by combining our own numerical results and results from the literature.

Sanjeevi and Padding [53] studied recently the flow past oblate spheroids. They observed that (8) and (9) obtained in the Stokes regime match well numerical results even in inertial 

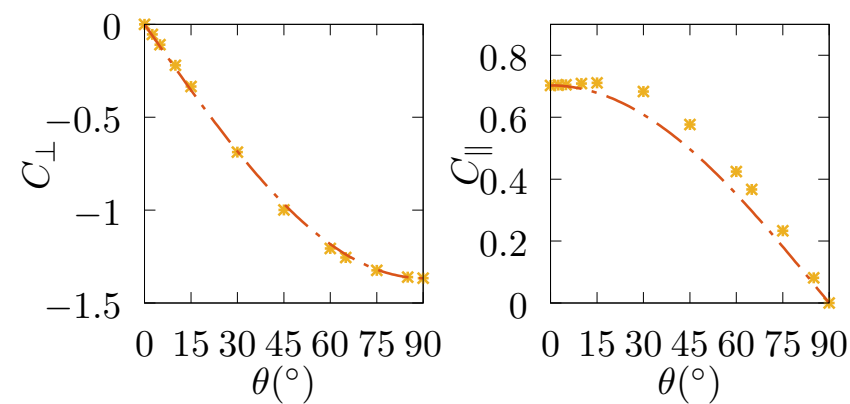

FIG. 28: Perpendicular and parallel force coefficients for $R e=50$ : * numerical results, $--(6)$ and $(7)$.

configurations $(R e=2000)$. Even if cylinders have a shape different from spheroids we may expect the same behaviour for cylinders and therefore a good agreement of (8) with our numerical results even in inertial regimes. This is verified in FIG. 28. The forces perpendicular and parallel to the flow direction fit very well (6) and (7) obtained in the Stokes regime. We note a very slight difference between (7) and our numerical results for $30^{\circ} \leq \theta \leq 60^{\circ}$.

The problem is that (8) and (9) require $C_{D \theta=0^{\circ}}$ which is a priori unknown. Results obtained using slender body theory $(L \gg D)$ (see for instance Batchelor [4]) can be used to obtain a rough estimate of $C_{D \theta=0^{\circ}}$. At the lowest order in $D / L$ the slender body theory gives [Note4]: $C_{D \theta=0^{\circ}} \approx 1 / 2 C_{D \theta=90^{\circ}}$. Thus for moderate Reynolds numbers $25 \leq R e \leq 100$ we suggest the following law for the drag:

$$
C_{D} \approx C_{D \theta=90^{\circ}}\left(\frac{1}{2} \cos ^{2} \theta+\sin ^{2} \theta\right)
$$

and the lift :

$$
C_{L y} \approx C_{L y \theta=90^{\circ}}\left(\frac{1}{2} \cos \theta \sin \theta-\sin \theta \cos \theta\right) .
$$

As can be seen in Fig. 29, laws (6) and (7) derived in the Stokes regime are not able to reproduce accurately the drag and lift forces exerted on the cylinder at the highest Reynold number $R e=250$. An important deviation is observed in the range $45^{\circ} \leq \theta \leq 75^{\circ}$ of $C_{\perp}$ and $15^{\circ} \leq \theta \leq 60^{\circ}$ for $C_{\|}$. We offer below simple geometrical arguments to modify the laws in order to give reasonable agreement in this regime. The basic idea is to keep the behaviour of the Stokes law when it works well (low $\theta$ for $C_{\perp}$ and high $\theta$ for $C_{\|}$) and change the law when departure manifests. The normal force on the cylinder for $\theta=90^{\circ}$ can be written as $F_{\perp \theta=90^{\circ}}=C_{\perp \theta=90^{\circ}}^{\text {bis }}(1 / 2) L D \rho U^{2}$ where the superscript bis is used to indicate that the normal force coefficient is obtained using the projected surface area rather than $L D$. The drag 

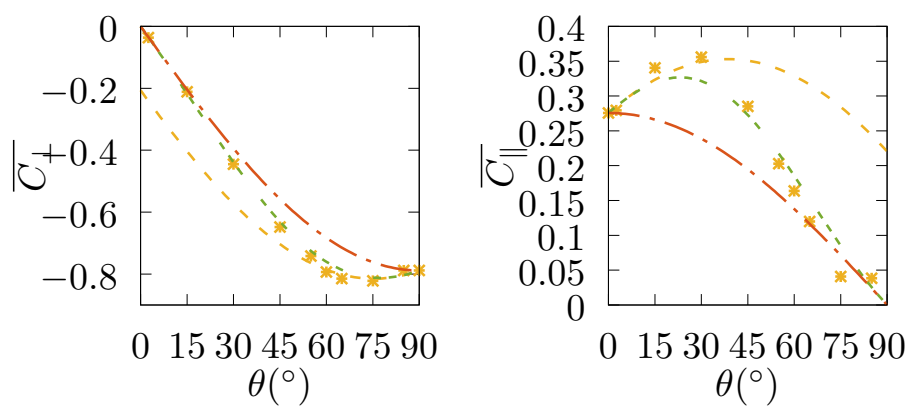

FIG. 29: Perpendicular and parallel force coefficients for $R e=250$ : * numerical results, $---(6)$ and (7), - - (17) and (18),--- (19) and (20).

force on a yawed cylinder can also be written as $F_{\perp \theta=90^{\circ}-\alpha}=C_{\perp \theta=90^{\circ}-\alpha}^{\text {bis }} S_{\text {proj }} 1 / 2 \rho U^{2}$ where $S_{\text {proj }}=\pi D^{2} / 4 \cos \theta+L D \sin \theta$ is the projected surface area of the cylinder along a plane normal to the streamwise direction and $\alpha$ is a parameter which is not necessary small. The main assumption of our model is $C_{\perp \theta=90-\alpha}^{\text {bis }} \approx C_{\perp \theta=90^{\circ}}^{\text {bis }}$. This assumption is based on the semi-empirical idea that for high Reynolds number flows hydrodynamic forces are mainly dominated by pressure, which can be roughly estimated using the projected surface area. Assuming that this is true, we get:

$$
C_{\perp} \approx C_{\perp \theta=90^{\circ}}\left(\frac{\pi}{4} \frac{D}{L} \cos \theta+\sin \theta\right)
$$

Using the same kind of argument, i.e., $C_{\| \theta=0+\alpha}^{\mathrm{bis}} \approx C_{\| \theta=0^{\circ}}^{\mathrm{bis}}$, a similar relationship can be obtained for the force coefficient parallel to the cylinder :

$$
C_{\|} \approx C_{\| \theta=0^{\circ}}(\cos \theta+A \sin \theta)
$$

where $A$ is a constant which should be equal to $\frac{4}{\pi} \frac{L}{D}$ following the formal derivation. In order to fit as well as possible our numerical results we use $A=0.8$ instead. From our numerical results, we note that $A$ should be a slightly increasing function of $R e$ but for the sake of simplicity $A$ is chosen constant. A key point when looking at (17) and (18) is that they contain terms similar to the equations derived in the Stokes regime while no assumption of this type has been made. As shown in FIG. 29, (17) and (18) match well our numerical results for high and low yaw angles, respectively. In order to obtain a solution uniformly valid on the whole range of $\theta,(17)$ and (18) are matched to equations (6) and (7). To this aim we use powers of trigonometric functions in order to make some terms of (17) and (18) negligible when the other terms approach the Stokes law and obtain: 


$$
\begin{gathered}
C_{\perp} \approx C_{\perp \theta=90^{\circ}} \sin \theta+C_{\perp \theta=90^{\circ}} \frac{\pi}{4} \frac{D}{L} \cos \theta \sin ^{2} \theta \\
C_{\|} \approx C_{\| \theta=0^{\circ}} \cos \theta+0.8 C_{\| \theta=0^{\circ}} \sin \theta \cos ^{2} \theta
\end{gathered}
$$

Even if the above equations contained terms similar to the terms in the Stokes law, they are different from equations obtained using a formal development in $R e$ in the Oseen regime [11]. This is not surprising since they have been derived using numerical simulations for Re $\gg 1$. (19) and (20) match pretty well our numerical results, as plotted in FIG. 29. Expressions for the drag coefficient and the lift coefficient can be obtained straightforwardly but we still need $C_{D \theta=0^{\circ}}$. Based on our numerical results we choose $C_{D \theta=0^{\circ}}=0.4 C_{D \theta=90^{\circ}}$. The resulting equations shown below are used for the highest Reynolds number $150 \leq R e \leq$ 250:

$$
\begin{gathered}
C_{D} \approx C_{D \theta=90^{\circ}}\left[0.4 \cos \theta\left(\cos \theta+0.8 \sin \theta \cos ^{2} \theta\right)+\sin \theta\left(\sin \theta+\frac{\pi}{4} \frac{D}{L} \cos \theta \sin ^{2} \theta\right)\right], \\
C_{L y} \approx C_{D \theta=90^{\circ}}\left[0.4 \sin \theta\left(\cos \theta+0.8 \sin \theta \cos ^{2} \theta\right)-\cos \theta\left(\sin \theta+\frac{\pi}{4} \frac{D}{L} \cos \theta \sin ^{2} \theta\right)\right] .
\end{gathered}
$$

At this stage several comments can be made. Laws (15) and (16) obtained using linearity of Stokes equations might be valid for other aspect ratios. Indeed the linearity of Stokes equations remains valid for all aspect ratios and these laws have proven to be valid even for spheroids of high aspect ratio [53]. The assumption $C_{D \theta=0^{\circ}} \approx 1 / 2 C_{D \theta=90^{\circ}}$ is also supposed to give more accurate results as $L / D$ increases [4]. However (21) and (22) obtained using a geometrical argument have no reason to be valid for other $L / D$.

Deriving a law for the torque (or pitching-torque since the angular velocity is zero) valid for high Reynolds number flows and arbitrary $L / D$ is beyond the scope of the present study. The reader interested in that question may consult Zastawny et al. [66]. However, physical arguments and a simple model are given in the following. The hydrodynamic torque reads $\mathbf{T}=\int_{S} \mathbf{r} \times \boldsymbol{\sigma} \cdot \mathbf{n} d S$ where $\mathbf{r}$ is the local position relative to the cylinder mass center. We define the hydrodynamic center $\mathbf{r}_{\mathbf{h c}}$ as the point where the hydrodynamic torque vanishes. The hydrodynamic torque can thus be written:

$$
\mathbf{T}=\mathbf{r}_{\mathbf{h c}} \times \mathbf{F}
$$


In the following, straightforward assumptions are made to derive a closed expression for the torque. We define a Cartesian coordinate system as $\left(x_{\|}, y_{\perp}, z\right)$ where $x_{\|}$is the axis of symmetry of the cylinder starting from its mass center, as illustrated in FIG. 27. Our main motivation is to find the $z$ component of the torque. From flow symmetry considerations, we assume that $\mathbf{r}_{\mathbf{h c}} \approx\left(x_{\| h c}, 0,0\right)$ which does not seem to be a strong assumption except in cases when $(x, y)$ planar symmetry is broken [Note5]. We obtain:

$$
T_{z} \approx x_{\| h c} F_{\perp}
$$

From (24) and assuming that we know the normal force exerting on the cylinder, the new unknown to derive an expression for the torque is the position of the hydrodynamic center $x_{\| h c}$. This equation supports the choice of $L$ as the characteristic length in the expression of $C_{T z} \cdot x_{\| h c}$ is made dimensionless by dividing by $L$ rather than $D$. With the help of (24), (10) derived for $R e \ll 1$ can be understood as follows: since to leading order in $R e$ the normal force varies as $\sin \theta$, the hydrodynamic center position $x_{\| h c}$ varies as $\cos \theta$. Two main questions remain to be addressed (i) is this law valid for higher Reynolds numbers ? and (ii) can we estimate the magnitude of the torque?

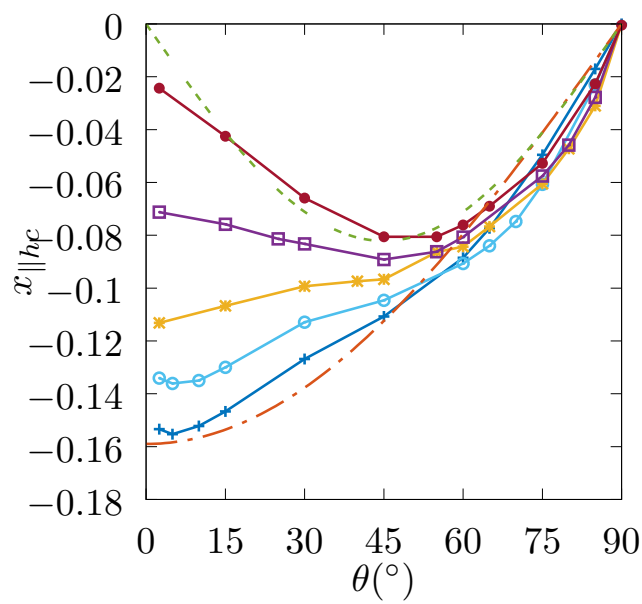

FIG. 30: Position of the hydrodynamic center normalized by the length as function of the yaw angle: $+R e=50, \circ R e=100, * R e=150, \square R e=200, \bullet R e=250,---(25)$ for

$$
R e=50,---(26) \text { for } R e=250 .
$$

FIG. 30 aims to give some answers to these questions. It plots the variation of $x_{\| h c}$ as a function of $\theta$ for different $R e$. The hydrodynamic center position is computed as: $x_{\| h c}=T_{z} / F_{\perp}$ which becomes singular when $\theta \rightarrow 0$. We note that $x_{\| h c}$ stays close to the 
cylinder mass center for all the configurations studied. For $R e=50$ and $R e=100, x_{\| h c}$ varies as $\cos \theta$. Thus, as for the force, the law (10) obtained in the Oseen regime remains

valid for moderate Reynolds number. Since the magnitude of $x_{\| h c}$ decreases linearly with Re for $\theta=45^{\circ}$, we suggest the following law for the hydrodynamic center position:

$$
x_{\| h c}=\sqrt{2}(a R e-b) \cos \theta .
$$

where $a=1 / 6600$ and $b=0.12$ are parameters fitted with our numerical results. This law is used for $25 \leq R e \leq 150$.

For $R e=200$ and $R e=250$, the magnitude of $x_{\| h c}$ decreases with decreasing $\theta$ for $\theta \leq 45^{\circ}$. (25) is not able to capture this behaviour. We thus suggest the following law:

$$
x_{\| h c}=2(a R e-b) \sin \theta \cos \theta
$$

which fits very well our numerical results for $R e=250$. Once again some comments can be made on the validity of these correlations. For $R e \ll 1$, calculations in the Oseen regime [11] predict a linear increase of the dimensional torque with $R e$ and a dimensional normal force constant to leading order in $R e$. Thus, to leading order in $R e, x_{\| h c}$ increases linearly with Re. This disagrees with (25) where the magnitude is seen to decrease with Re. This needs further investigation. We would like to point out that (26) should be taken cautiously since it tends to zero for $R e \approx 792$, which clearly disagrees with previous observations on the flow past a cylinder at high Reynolds number [27].

\section{Numerical results}

Moderate $R e(25 \leq R e \leq 100) \quad$ Figs. 31abde exhibit a highly satisfactory agreement between the predictions obtained in the Stokes regime with (8) and (9) and our numerical results for both the lift coefficient and the drag coefficient at $R e=25$ and $R e=50$. The semi empirical relations based on linear laws (15) and (16) also provide a good agreement with our numerical results. As observed by Sanjeevi and Padding [53] for spheroidal particles, the laws derived by assuming the linearity of Stokes equations match closely numerical results even for inertial regimes. For $R e=25$ and $R e=50$, the predictions of both the lift coefficient and the drag coefficient given by the semi empirical relation of Rosendahl [50] are less accurate than the laws based on the linearity of Stokes equations. 

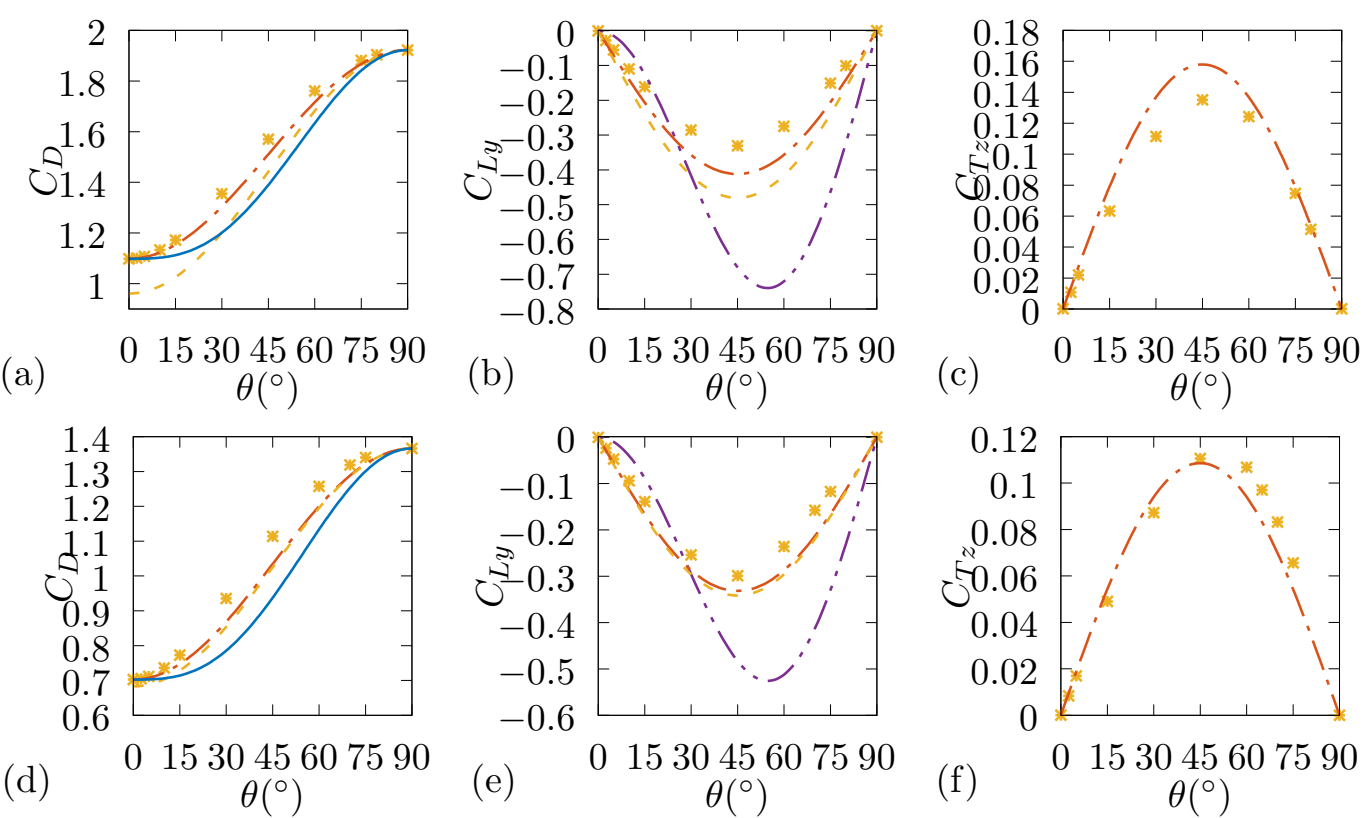

(c)
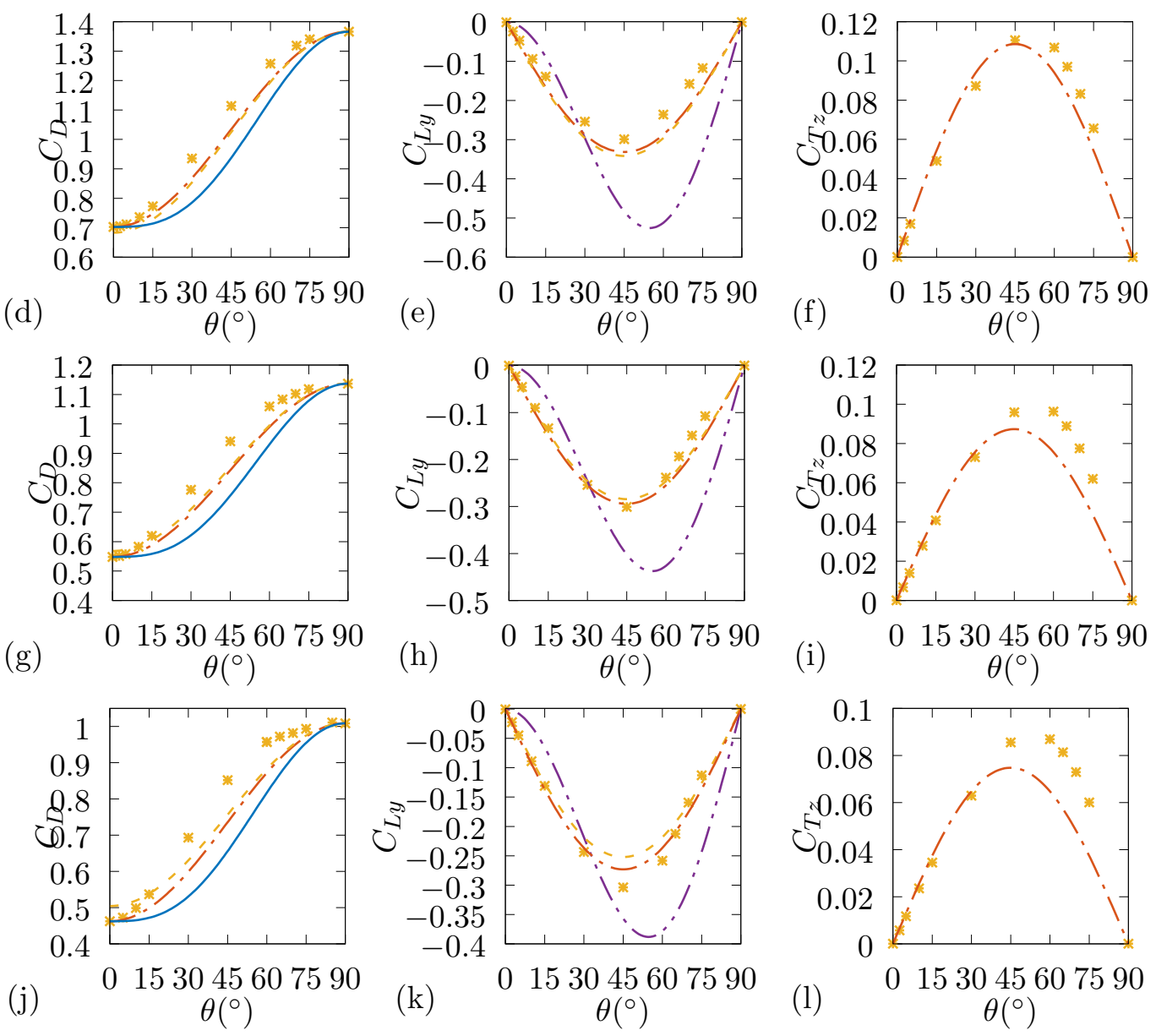

FIG. 31: Drag, lift and torque coefficients for: a-c $R e=25$, d-f $R e=50, \mathrm{~g}-\mathrm{i} R e=75, \mathrm{j}-\mathrm{l}$ $R e=100 . *$ numerical results, - empirical relation (14) of Rosendahl [50], --- (8) and

(9) for the force / (24) based on (25) and (6) for the torque, --- - (15) and (16).

As Re increases to 75 and 100, the drag force plotted in Fig. 31gh and Fig. 31jk, respectively, slowly departs from the predictions obtained in the Stokes regime. Indeed the drag coefficient is underestimated by (8) and (15), especially for $\theta \in\left[30^{\circ}, 75^{\circ}\right]$. However the predictions for the lift coefficient given by the laws derived by assuming the linearity of Stokes equations still agree very well with our numerical results. 
Fig. 31c, Fig. 31f, Fig. 31i and Fig. 311 show the torque coefficient for $R e=25$, $R e=$ 50, $R e=75$ and $R e=100$, respectively, and compare the law (24) derived by assuming a Oseen regime (based on (25) and (6), [11]) to our numerical results. The torque is zero for $\theta=0^{\circ}$ and $\theta=90^{\circ}$ but the only stable configuration is $\theta=90^{\circ}$ as explained by Khayat and Cox [30]. The agreement between our numerical results and (24) based on (25) and (6) is satisfactory even if our numerical results plot is slightly shifted to higher values for high $\theta$ when increasing $R e$. We also note that for $R e=25$, (24) based on (25) and (6) slightly overestimates the maximum torque. This overestimation is attributed to an overestimation of the position of the hydrodynamic center $x_{\| h c}$. For $R e=75$ and $R e=100$, (24) based on (25) and (6) slightly underpredicts the maximum torque. This underestimation is here attributed to an underprediction of the normal force $F_{\perp}$.
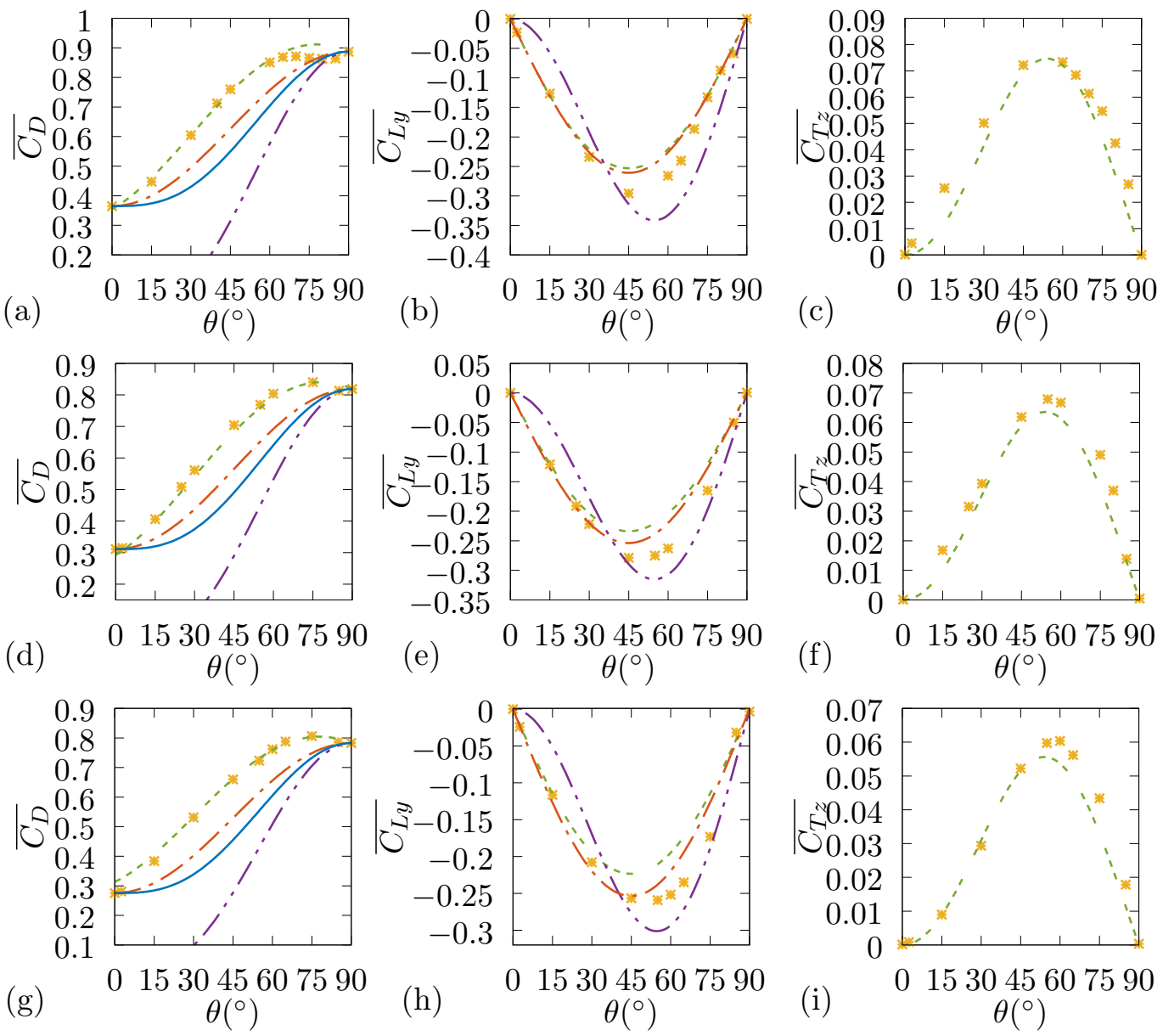

FIG. 32: Drag, lift and torque coefficients for: a-c $R e=150$, d-f $R e=200$, g-i $R e=250$. * numerical results, - empirical relation (14) of Rosendahl [50], --_ (8) and (9), --- IP, --- (21) and (22) for the force / (24) based on (26) and (19) for the torque. 
High Re $(100<R e \leq 250) \quad$ FIGS. 32adg clearly evidence that the IP in its original form given by (12) does not fit well our numerical results for the drag coefficient since the drag coefficient when $\theta=0^{\circ}$, i.e., $C_{D \theta=0^{\circ}}$, is not taken into account. Taking into account $C_{D \theta=0^{\circ}}$ leads to (14) that exhibits a better agreement. Marked differences in the range $30^{\circ} \leq \theta \leq 60^{\circ}$ are still noticeable though. For all $\theta$, the linear law yields a more satisfactory match with our numerical results than the IP and its modification due to Rosendahl [50]. The law (21) based on geometric arguments is the law that gives the best agreement for the drag force for $R e=150,200$ and 250. In the limit of high $\theta$, we do note a slight departure of this law from our numerical results. For $R e=150$ the drag coefficient is almost constant for $75^{\circ} \leq \theta \leq 90^{\circ}$ while $(21)$ predicts a slight increase of $\overline{C_{D}}$ from $90^{\circ}$ to $70^{\circ}$ degrees. However, this is a minor discrepancy.

The agreement between the IP and our numerical results is better for the lift coefficienty, as illustrated in Figs. 32beh, than for the drag coefficient. The IP gives results of comparable accuracy to (9). The law (22) based on geometric arguments slightly underpredicts the lift coefficient. This is due to the fact that (19) overpredicts the parallel force for $50^{\circ} \leq \theta \leq 80^{\circ}$.

The torque coefficient is still well approximated by (24) based on (26) and (19) even if we observe that the maximum of $C_{T z}$ is slightly underpredicted for the three values of $R e=150,200$ and 250. This underprediction is attributed to an underprediction of the hydrodynamic center position $x_{\| h c}$.

General outcome and further comments on force and torque laws The overall picture of force and torque laws is as follows. The laws obtained in the Stokes regime or the Oseen regime for the drag and lift coefficients give a good agreement for $R e \leq 100$. At higher Reynolds numbers (21) and (22) based on geometric arguments should be preferred. The torque is well approximated by (24), provided the normal force and the hydrodynamic center position are properly modelled as function of $R e$. The value of $\theta$ for which the torque is maximal increases from $45^{\circ}$ to $55^{\circ}$ when Re increases.

FIGS. 31 and 32 also show that $C_{D \theta=90^{\circ}}$ and $C_{D \theta=0^{\circ}}$ decrease significantly as $R e$ increases as $R e^{-1 / 2}$ (not shown here). This is in line with the scaling of the viscous stress in the boundary layer approximation [5]. However the maximum lift force as a function of $\theta$ remains approximatively constant as $R e$ increases. This kind of behaviour has also been observed for the lift force exerted on a sphere immersed in a linear shear flow Kurose and 
Komori [32].

\section{CONCLUSION}

We investigated the inertial flow past a 3D cylinder of aspect ratio 3 by particle-resolved simulation. The ranges of Reynolds number $R e$ and yaw angle $\theta$ examined are [25, 250] and $\left[0^{\circ}, 90^{\circ}\right]$, respectively. We analysed the wake structure and the hydrodynamic force and torque exerted on the cylinder. Ten different regimes and corresponding wake patterns were observed depending on $\theta$ and Re spanning standing toroidal eddy, one or two steady counterrotating vortex pairs, periodic shedding of single-sided hairpin vortices, quasi-symmetric shedding of double-sided vortices nicknamed accordion folds, periodic shedding of one or two pairs of counter-rotating vortices, and unsteady shedding of asymmetric double-sided hairpin vortices. When the cylinder is perpendicular to the flow direction, we confirm the transition scenario observed by Inoue and Sakuragi [25].

We plotted the hydrodynamic force and torque as a function of $R e$ and $\theta$ and attempted to fit these plots with force and torque correlations. This is an important result of this study as these correlations are meant to be later used in higher scale models as, e.g., Euler/Lagrange and Euler/Euler models, for the simulation of dilute suspensions of cylinders. The correlation given by Rosendahl [50] based on the IP is shown to be poorly accurate to describe the drag force exerted on a yawed cylinder. For the aspect ratio $L / D=3$ studied and $25 \leqslant R e \leqslant 100$, a law derived in the Stokes regime seems to be better suited. We proposed simple laws derived in the Stokes regime that do not explicitly require the drag coefficient when the cylinder is aligned with the flow direction. These laws give reasonable agreement with numerical results for both the drag force and the lift force. The IP is also shown to be poorly accurate to describe the drag force on a yawed cylinder for $100 \leqslant R e \leqslant 200$. Instead, we derived an empirical relationship based on geometrical consideration that gives better agreement with our numerical results. Based on a symmetry assumption, we also derived a law for the torque that explicitly involves the normal force. For all Re examined, our torque law matches well our numerical results as long as we can predict properly the position of the hydrodynamic center. We showed that this can done using a fit that involves trigonometric functions. Based on the results of Cox [11] and our numerical results, we suggested 2 different laws in the ranges $R e \in[25,150]$ and $R e \in[150,250]$, respectively. The resulting predictions 
of these 2 laws give full satisfaction.

The range of validity of the IP as a function of $L / D$ needs further investigation. Indeed for very high $R e$ and $L / D$, the IP supplies accurate predictions [20,69]. We can hence redo a similar study for higher $L / D$ if we wish to further challenge the IP. Therefore the flow map shown in FIG. 3 would be enriched with a third parameter, the aspect ratio $L / D$. This would also give us the opportunity to compare our numerical results to the results of Ramberg [47]. The specific configuration of the cylinder aligned with the flow also deserves further investigation, first to provide a lower bound for the drag coefficient, second to investigate the impact of $L / D$ on boundary layer detachment and third to study the bifurcation scenario. For instance, Ern et al. [14] gave a threshold value for the Reynolds number $R e_{c}$ associated with the first bifurcation for a disk $L / D \leq 1$ aligned with the flow. It is estimated as $R e_{c}=116.5(1+L / D)$.

Finally, we intend to investigate the problem of a freely moving 3D cylinder as, e.g., a heavy cylinder settling in a quiescent light fluid. We already performed such a study for angular polyhedra in Rahmani and Wachs [46]. Our numerical method is actually better suited to moving particles than to flows past fixed obstacles (see [15, 46, 61] among other references), although it can be used (as here) for the flow past a single or multiple obstacles [13]. While improvements to our numerical method are always suitable (higher order schemes, enhanced parallel performance), particle-resolved simulations are computationally demanding. The finest mesh selected in this work comprised 100 millions of grid cells. The corresponding computation ran for several days/weeks on 512 cores. However, as shown in this paper, inertial regimes lead to thin boundary layers around freely moving or fixed particles that need to be captured properly. This puts some stress on the mesh size, which consequently increases the number of cells, in particular in a constant grid size approach as ours. An alternative would be to re-develop our Fictitious Domain method in an octree/adaptive mesh refinement [44] framework that would give us the ability to refine the mesh wherever required and to loose it away from solid particles and/or from regions of the flow with strong velocity gradients.

Acknowledgements This work was granted access to the HPC resources of GENCI under the allocation c20162a7728 and c20172a7728. JLP wish to thank Jacques Magnaudet for fruitful discussions regarding this subject. 


\section{Distributing Lagrangian points at the surface of a cylinder}

Distributing points as uniformly as possible on a random surface is a complicated task even for simple geometries. A way to solve this problem is to treat each point as a repulsive particle and solve dynamic simulations until the system reaches a minimum of repulsive energy [51]. While being accurate this method can be very costly when dealing with a large number of particles. Another way to distribute points evenly on spheres is to use spiralling distributions [51, 63]. This method is not fully rigorous but it gives a relatively uniform distribution designed for isotropic hydrodynamic computations independent of the particle orientation. The method presented here means to be computationally cheap and accurate.

In order to obtain an accurate hydrodynamic solution over the cylinder it is necessary to describe the corners as precisely as possible. $N_{c}$ Lagrangian points are distributed on the circle such that $N_{c}=\left\lfloor\pi D / l_{p}\right\rfloor$ where $\lfloor a\rfloor$ is the floor of the real number $a$ and $l_{p} \approx \sqrt{3} \Delta x$ is the desired distance between each lagrangian point. Doing so, owing to the finite curvature of the circle, an error of order $\mathcal{O}\left(l_{p}^{2}\right)=\mathcal{O}\left(\Delta x^{2}\right)$ is introduced for the distance between each points. Mapping the cylinder length can also be done in a straightforward manner. Noting that this part can be roll out, the resulting rectangular domain is mapped with a diamond mesh (FIG. 33a). The lateral distance between each point along the length of the rectangle is $\sqrt{3} / 2 l_{p}$ while the width distance is $l_{p}$. Owing again to the finite curvature of the cylinder an error on the distance of order $\mathcal{O}\left(\Delta x^{2}\right)$ is made.

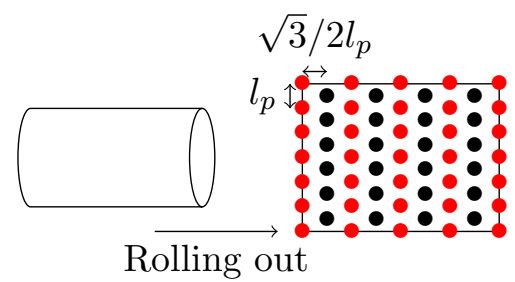

(a)

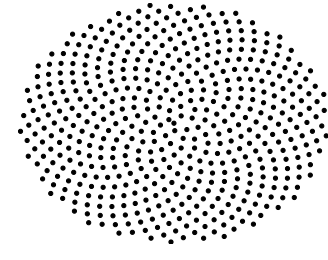

(b)

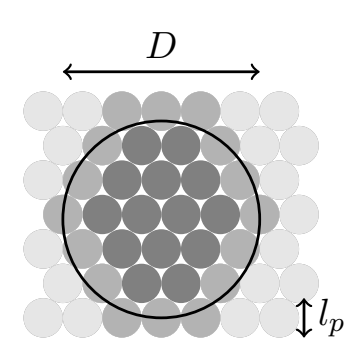

(c)

FIG. 33: (a) Mapping of the cylinder length with a diamond mesh. (b) Distribution of 512 points given by Vogel's method [60]. (c) Hexagonal distribution of circles in a disk. Black circles are the one contained entirely in the disk, dark circle those intersecting the disk and grey circle exterior one. 
Distribute points uniformly on the surface of the disks appears much more challenging than distributing it on the main part of the cylinder. A natural way which follows directly the distribution of points on the corner of the particle is to distribute points in the disk as concentric circle. However this distribution is far from uniform. Indeed it highly depends on the position of the first point chosen when computing the circles and the distance between each concentric circles. A more sophisticated approach comes from the distribution of individual flowers in flowers head [60]. The main idea is to distribute the points following a Fermat spiral whose equations in polar coordinate is written: $r=\left(1-l_{p} / D\right) \sqrt{i / N}$, $\phi=\pi(3-\sqrt{5}) i$ where $r$ is the distance from the circle center, $i$ the index number of the points, $N$ the total number of points, $\phi$ the angle and $\pi(3-\sqrt{5})$ the golden number. FiG. 33b displays this distribution. The choice of the golden number as a parameter in $\phi$ is not trifling and correspond to the angle for which the particles are the most uniformly distributed for a given set of spiralling distribution [49]. This model is not closed since it requires the number of points $N$ to be distributed on the disk. This number is closely linked to the densest packing of equal circles of diameter $l_{p}$ in a larger circle of diameter $D$. This problem is only solved for a limited number of circles. In the following a simple approximation for the maximum number of circle that can be packed in one bigger circle is presented.

For a two dimensional packing of equal-size circles the density $\eta$ is defines as the ratio between the solid surface and the total surface. In the case of an hexagonal arrangement (which can be shown to be the highest density arrangement), the density is $\eta_{h}=\pi /(2 \sqrt{3})$. This result can be found taking three circles in contact in FIG. 33c and calculating the ratio between the area of the circles contained in the equilateral triangle formed with their center and the area of the triangle. For $N \gg 1$ the maximum packing in the disk $\left(\eta_{m}\right)$ is assumed to be very closed to an hexagonal arrangement as illustrated in FIG. 33c. Thus we have :

$$
\eta_{m} \approx \eta_{h}-\frac{N_{i} \pi l_{p}^{2} / 4}{\pi D^{2} / 4},
$$

where $N_{i}$ is the number of sphere intersecting the outer circle (in dark in FIG. 33c). Since $N_{i} \approx \frac{\pi D}{l_{p}}$ and $\eta_{h} \pi\left(D-l_{p}\right)^{2} / 4 \approx N \pi l_{p}^{2} / 4$, a relationship between the actual density and the number of circle can be obtained:

$$
\eta_{m} \approx \eta_{h}-\frac{1}{\sqrt{N / \eta_{h}}+1} .
$$

FIG. 34 shows the very good agreement between equation 28 and the exact results for the densest packing obtained numerically. 


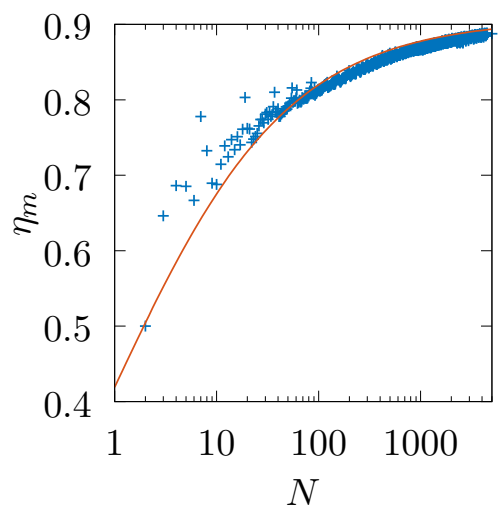

FIG. 34: Maximum density for equal circle contained in a bigger circle. Points are exact value calculated numerically from http://hydra.nat.uni-magdeburg.de/packing/cci/. The red line represents the maximal packing obtained from equation 28.

Since by definition $\eta_{m}=N l_{p}^{2} / D^{2}$ we obtain an equation for the maximal number of particles of diameter $l_{p}$ contained in a disk of diameter $D$ :

$$
N \approx \frac{D^{2}}{l_{p}^{2}}\left(\eta_{h}-\frac{1}{\sqrt{N / \eta_{h}}+1}\right)
$$

Equation 29 is solved with a fixed point algorithm. The difference between two consecutive solutions is less than one percent in 3-4 iterations.

\section{$L / D=1, \theta=0^{\circ}:$ results with a boundary-fitted method}

\section{Franck Auguste, David Fabre, Jacques Magnaudet}

In this appendix the flow past a $\theta=0^{\circ}, L / D=1$ cylinder is investigated. After a short presentation of the numerical methods used for the study, the different hydrodynamic regimes encountered are presented.

\section{Numerical method and variables definition}

The JADIM code developed at IMFT is employed. Since, the numerical method has been detailed in previous articles $[3,16,33]$ it will only be summarized here. The 3D Navier-Stokes equations for an incompressible fluid are solved on an orthogonal curvilinear

mesh which follows closely the boundaries of the body (Boundary-Fitted Method). The 
equations are discretized using finite volume method and centered schemes which ensure second-order space accuracy. The time step advancement strategy is a mixed Runge-Kutta / Cranck-Nicholson algorithm. The incompressibility condition is satisfied at the end of each time step thanks to a projection method.

The computations are performed in a cylindrical domain. The grid resolution is $160(x) \times$ $75(r) \times 32(\phi)$ nodes, where $x$ is the distance on the cylinder symmetry axis, $r$ the radial distance from this axis and $\phi$ the azimuthal angle. A non-uniform spatial distribution is used to properly capture the body wake and the boundary layer developed near the solid surface. The characteristic cells size is $\approx 0.01 D$ near the body and $\approx 0.1 D$ in the near wake localized by $x \approx 2 D$ ( $x=0$ is the location of the cylinder centroid). The size of the domain is $10 D$ (resp. 20D) in lateral and upstream (resp. downstream) directions. Symmetry boundary conditions is imposed on the lateral wall, while inlet and outlet boundary conditions are imposed on the upstream and downstream boundary respectively.

The influence of the Reynolds number is studied over the range [20:460] by step of 10 in most of the interval. This implies a non-negligible incertitude on the proposed threshold values associated to the nature of the wake instabilities. Simulations run during $\mathcal{O}\left(10^{2}\right) D U^{-1}$ physical time to achieve the instability growth and saturation. The normalization of the

physical parameters $(U, \omega)$ is the same as in the main body of the paper. The pressure is normalized by $\rho U^{2}$, the vorticity by $U / D$, the drag and lift force by $1 / 2 \rho U^{2} D^{2}$ and the Strouhal number is defined as $S t=\frac{f D}{U}$ where $f$ is the frequency of vortex shedding. A convenient rotation is applied on the numerical solution showing a symmetry plane in such a way that the $(x, y)$ plane becomes the symmetry plane.

\section{Axisymmetric solution}

As observed at low Re in the wake of sphere or disk [65], the flow developed around the $L / D=1$ thick cylinder is a steady axisymmetric solution (invariance per $x$-rotation). Except for very low $R e \lesssim 2.5[2]$, the body wake is characterized by a toroidal region for which the recirculating length increases with Re (see Section). FIG. 35 illustrates this hydrodynamic regime $(R e=200)$ showing the pressure contours and the streamlines.

A significant difference with respect to the sphere and disk wake is the appearance of a detachment at the upstream ridge for $R e \gtrsim 205$. This detachment induces a new eddy 


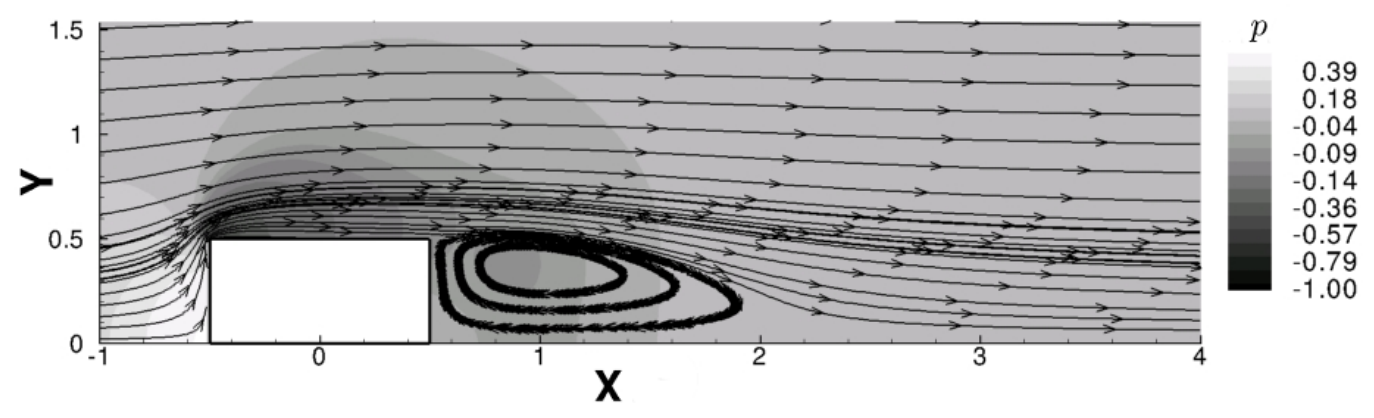

FIG. 35: $R e=200$ : pressure contours and streamlines in an arbitrary azimuthal plane.

structure with a torus shape, growing in size and intensity with $R e$ [2]. This regime is observed until $R e \approx 278$.

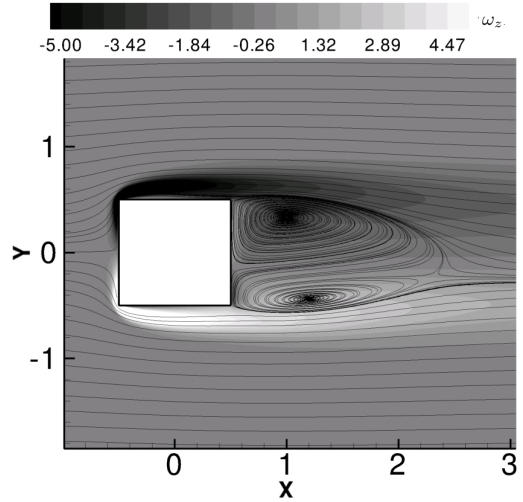

(a)

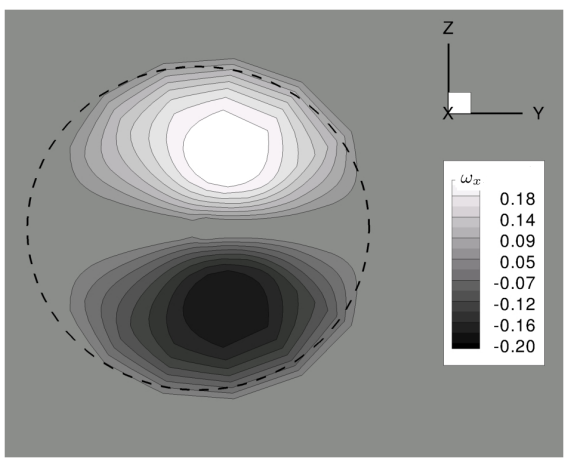

(b)

FIG. 36: $R e=290$ : (a) $\omega_{z}$ contours and streamlines in the symmetry plane $(z$ is the direction perpendicular to the symmetry plane); (b) longitudinal vorticity $\omega_{x}$ contours in a longitudinal plane localized one diameter past the rear body surface.

\section{Two counter-rotating vortices}

$R e \approx 278$ is the critical value for which the axisymmetric mode becomes unstable and the axial symmetry breaks (this critical value is estimated from the positive and negative instability growth rates near the bifurcation). However the observed regime remains steady. The bifurcation occurring at $R e \approx 278$ is thus a steady one. No hysteresis is found. This regime is characterized by a symmetry plane as illustrated in FIG. 36a and Fig. 36b. A pair 
of counter-rotating vortices (bifid wake) appear in the wake and induce a transverse force $\left(C_{L} \neq 0\right)$.

\section{Vortex shedding}

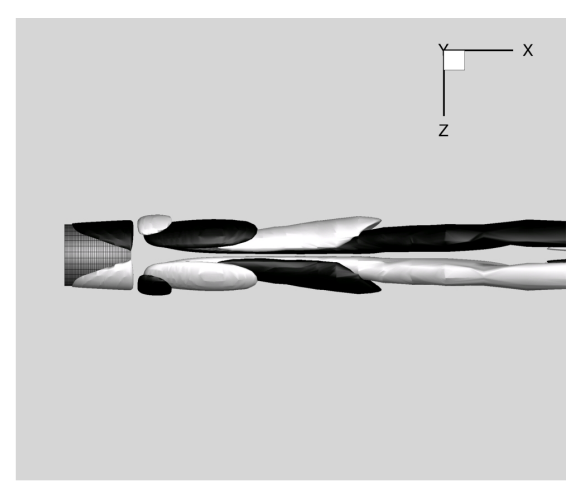

(a)

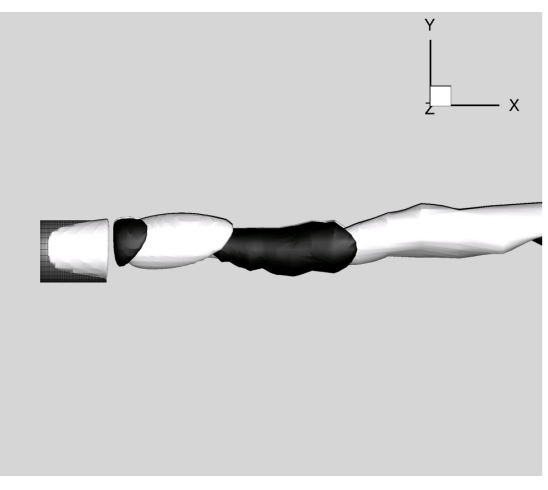

(b)

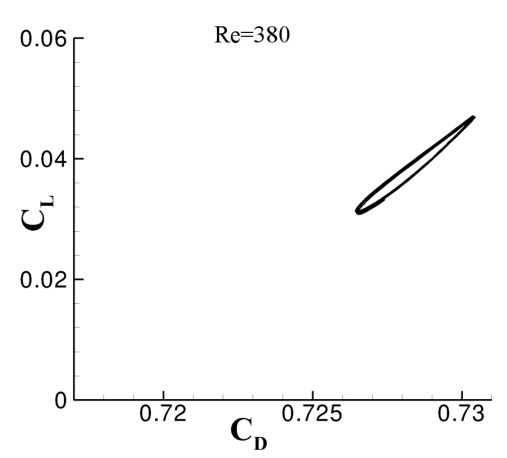

(c)

FIG. 37: $R e=380$ : (a resp. b) $\omega_{x} \pm 0.25$ isosurfaces with a view parallel (resp. perpendicular) to the symmetry plane; (c) force coefficients diagram

The next bifurcation appears for $R e \approx 355$ with the appearance of an unsteady mode. No hysteresis is found. Figures FIG. 37ab displays the detachment and advection of hairpins vortices in the $L / D=1$ wake $(R e=380)$. The wake is still symmetric with respect to the $(x, y)$ plane.

Discrete Fourier Transform (DFT) of the lift force indicates a unique unsteady mode associated to the dimensionless frequency $S t \approx 0.12$. Note that this value is comparable to the one found for the flow past a sphere (resp. disk) for the same wake regime : $S t_{s p h} \sim$ 0.13 (resp. $S t_{d i s k} \approx 0.12$ ). The force coefficients diagram $\left(C_{D} ; C_{L}\right)$ is plotted in FIG. 37c $(R e=380)$ and shows a loop attractor; the drag force oscillates with the same frequency as the lift force.

\section{The route to chaos}

For $R e \gtrsim 395$ a second frequency appears and the $\left(C_{D} ; C_{L}\right)$ attractor presents erratic loops as shown in Fig. 37a $(R e=400)$. The newly detected frequency is not an harmonic 
of $f$ and its value in dimensionless form approaches $\frac{1}{4} S t(R e=400) \approx 0.03$. The symmetry plane is still preserved up to $R e \approx 420$.

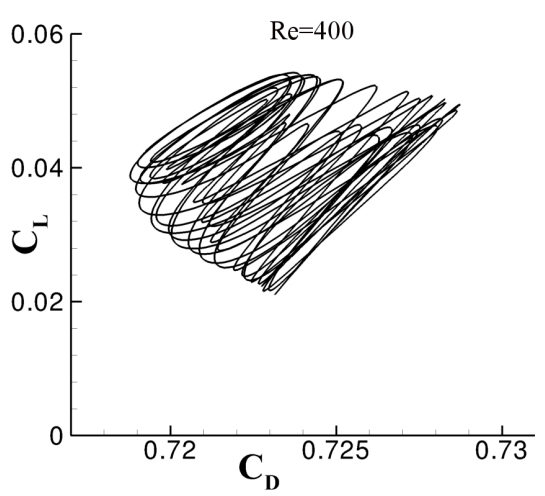

(a)

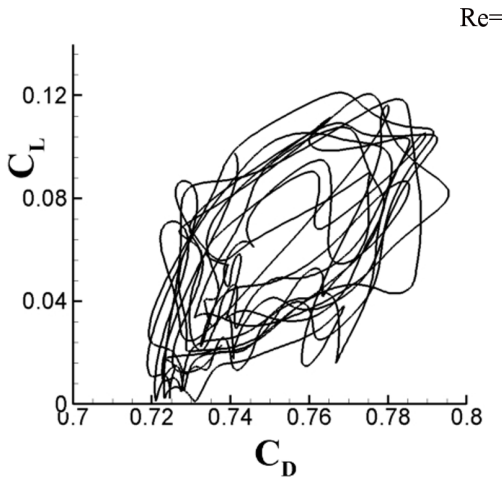

(b)

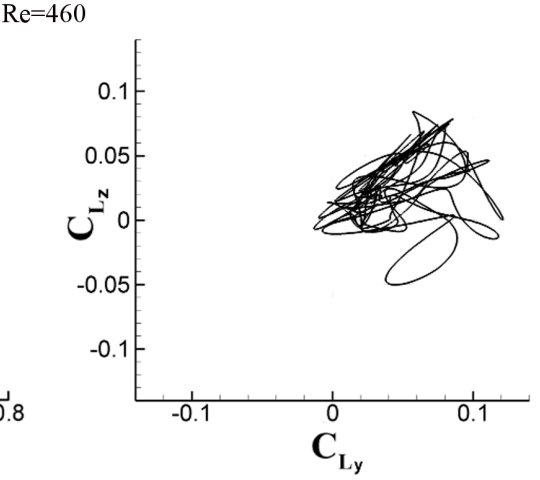

(c)

FIG. 38: (a) (resp. b) drag versus lift coefficient for $R e=400$ (resp. 460); (c) transverse forces coefficient diagram for $R e=460$.

This symmetry is distinctly broken for $R e \approx 450$ and the flow exhibits $3 \mathrm{D}$ patterns. FIG. 38b displays the hydrodynamic forces $\left(C_{L}=\sqrt{C_{L y}^{2}+C_{L z}^{2}}\right)$ observed at $R e=460$. The oscillation amplitude and the mean value of the lift force are significantly larger than the one encountered in the previous regime. The forces diagram FiG. 38c illustrates the chaotic character of the observed solution. The amplitude of the unsteady mode associated to secondary frequency defined in the previous section becomes predominant and leads to $\operatorname{St}(\operatorname{Re}=460) \approx 0.03$.

\section{Scenario of the first bifurcations}

FIG. 39 summarizes the non-3D chaotic regimes and associated bifurcations for $R e \leq 420$. The four encountered regimes (axisymmetric and bifid wakes, two vortex shedding types) are referenced by order of appearance as function of $R e$ as $R[1: 4]$. The signature of the unsteady character is weakly (resp. highly) visible on $C_{D}\left(\right.$ resp. $\left.C_{L}\right)$. The bifurcations scenario of the $L / D=1$ thick cylinder appears to be close to the sphere's one [2, 28, 34]. The nature of the fourth bifurcations and the route to chaos is found to be identical for each solid body. Following the bifurcations theory [12] applied to wake instabilities [16], the first (resp. second) bifurcation is as a pitchfork (resp. Hopf) regular one. This similarity is reinforced 


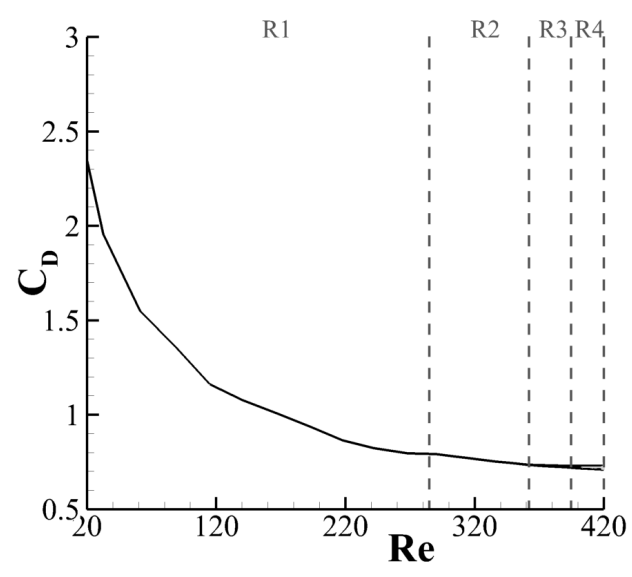

(a)

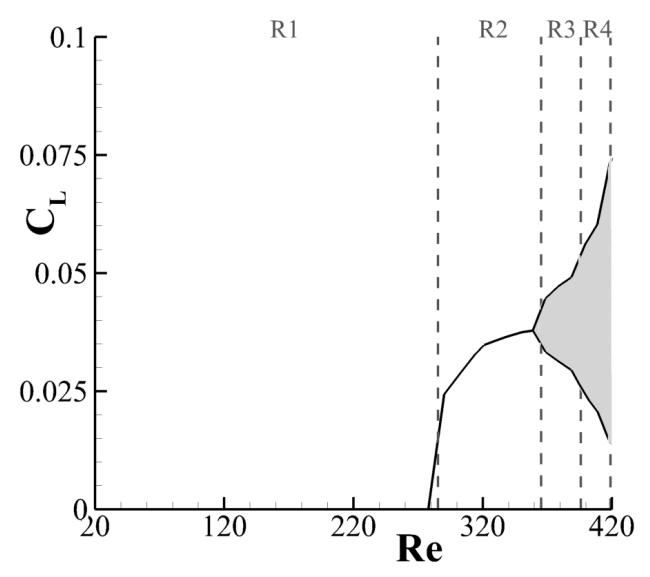

(b)

FIG. 39: $20 \leq R e \leq 420$ : (a resp. b) evolution of the drag (resp. lift force) coefficient with the Reynolds number. $R[1: 4]$ defines the four encountered wake regimes: axisymmetric, bifid wakes and the two unsteady regimes with one or more characteristic frequencies. The upper and lower values of the force coefficients are indicated with the black curves.

by the fact that the ratio between the critical thresholds for which each bifurcation occurs for flow past a sphere and the one in our situation is closed to be a constant: $\frac{R e_{s p h}}{R e_{L / D=1}}(R 1 \leftrightarrow$ $R 2) \approx \frac{R e_{s p h}}{R e_{L / D=1}}(R 2 \leftrightarrow R 3) \approx \frac{R e_{s p h}}{R e_{L / D=1}}(R 3 \leftrightarrow R 4) \sim[0.76: 0.81]$

* jean-lou.pierson@ifpen.fr

[1] Ardekani, M. N., Costa, P., Breugem, W. P., and Brandt, L. (2016). Numerical study of the sedimentation of spheroidal particles. International Journal of Multiphase Flow, 87:16-34.

[2] Auguste, F. (2010). Instabilités de sillage générées derrière un corps solide cylindrique, fixe ou mobile dans un fluide visqueux. These de doctorat.

[3] Auguste, F., Fabre, D., and Magnaudet, J. (2010). Bifurcations in the wake of a thick circular disk. Theoretical and Computational Fluid Dynamics, 24(1):305-313.

[4] Batchelor, G. (1970). Slender-body theory for particles of arbitrary cross-section in stokes flow. Journal of Fluid Mechanics, 44(3):419-440.

[5] Batchelor, G. K. (1967). An introduction to fluid dynamics. Cambridge university press. 
[6] Bernard, M., Climent, E., and Wachs, A. (2016). Controlling the quality of two-way euler/lagrange numerical modeling of bubbling and spouted fluidized beds dynamics. Industrial E Engineering Chemistry Research.

[7] Capecelatro, J. and Desjardins, O. (2013). An euler-lagrange strategy for simulating particleladen flows. Journal of Computational Physics, 238:1-31.

[8] Chrust, M., Bouchet, G., and DUŠEK, J. (2010). Parametric study of the transition in the wake of oblate spheroids and flat cylinders. Journal of Fluid Mechanics, 665:199-208.

[9] Clift, R., Grace, J., and Weber, M. (1978). Bubbles, drops, and particles.

[10] Coutanceau, M. and Bouard, R. (1977). Experimental determination of the main features of the viscous flow in the wake of a circular cylinder in uniform translation. part 1. steady flow. Journal of Fluid Mechanics, 79(2):231-256.

[11] Cox, R. (1965). The steady motion of a particle of arbitrary shape at small reynolds numbers. Journal of Fluid Mechanics, 23(4):625-643.

[12] Crawford, J. D. and Knobloch, E. (1991). Symmetry and symmetry-breaking bifurcations in fluid dynamics. Annual Review of Fluid Mechanics, 23(1):341-387.

[13] Dorai, F., Moura Teixeira, C., Rolland, M., Climent, E., Marcoux, M., and Wachs, A. (2015). Fully resolved simulations of the flow through a packed bed of cylinders: Effect of size distribution. Chemical Engineering Science, 129:180-192.

[14] Ern, P., Risso, F., Fabre, D., and Magnaudet, J. (2012). Wake-induced oscillatory paths of bodies freely rising or falling in fluids. Annual Review of Fluid Mechanics, 44:97-121.

[15] Esteghamatian, A., Hammouti, A., Lance, M., and Wachs, A. (2017). Particle resolved simulations of liquid/solid and gas/solid fluidized beds. Physics of Fluids, 29(3):033302.

[16] Fabre, D., Auguste, F., and Magnaudet, J. (2008). Bifurcations and symmetry breaking in the wake of axisymmetric bodies. Physics of Fluids, 20(5):051702.

[17] Fiechter, M. (1966). Vortex systems on slender rotating bodies and their effect on the aerodynamic coefficients. NASA technical memorandum.

[18] Ghidersa, B. and Dusek, J. (2000). Breaking of axisymmetry and onset of unsteadiness in the wake of a sphere. Journal of Fluid Mechanics, 423:33-69.

[19] Glowinski, R., Pan, T., Hesla, T., and Joseph, D. (1999). A distributed Lagrange multiplier/fictitious domain method for particulate flows. International Journal of Multiphase Flow, 25(5):755-794. 
[20] Hoerner, S. F. (1965). Fluid-dynamic drag: practical information on aerodynamic drag and hydrodynamic resistance. Hoerner Fluid Dynamics Midland Park, NJ.

[21] Hölzer, A. and Sommerfeld, M. (2008). New simple correlation formula for the drag coefficient of non-spherical particles. Powder Technology, 184(3):361-365.

[22] Hölzer, A. and Sommerfeld, M. (2009). Lattice boltzmann simulations to determine drag, lift and torque acting on non-spherical particles. Computers $\&$ Fluids, 38(3):572-589.

[23] Hu, H. H., Joseph, D. D., and Crochet, M. J. (1992). Direct simulation of fluid particle motions. Theoretical and Computational Fluid Dynamics, 3:285-306.

[24] Hunt, J. C., Wray, A. A., and Moin, P. (1988). Eddies, streams, and convergence zones in turbulent flows.

[25] Inoue, O. and Sakuragi, A. (2008). Vortex shedding from a circular cylinder of finite length at low reynolds numbers. Physics of Fluids, 20(3):033601.

[26] Jackson, R. (2000). The dynamics of fluidized particles. Cambridge University Press.

[27] Jayaweera, K. and Mason, B. (1965). The behaviour of freely falling cylinders and cones in a viscous fluid. Journal of Fluid Mechanics, 22(4):709-720.

[28] Johnson, T. and Patel, V. (1999). Flow past a sphere up to a reynolds number of 300. Journal of Fluid Mechanics, 378:19-70.

[29] Kempe, T. and Fröhlich, J. (2012). An improved immersed boundary method with direct forcing for the simulation of particle laden flows. Journal of Computational Physics, 231(9):36633684 .

[30] Khayat, R. and Cox, R. (1989). Inertia effects on the motion of long slender bodies. Journal of Fluid Mechanics, 209:435-462.

[31] Kim, I. and Elghobashi, S. (1998). On the equation for spherical-particle motion: effect of reynolds and acceleration numbers. Journal of Fluid Mechanics, 367(1):221-253.

[32] Kurose, R. and Komori, S. (1999). Drag and lift forces on a rotating sphere in a linear shear flow. Journal of fluid mechanics, 384:183-206.

[33] Magnaudet, J., Rivero, M., and Fabre, J. (1995). Accelerated flows past a rigid sphere or a spherical bubble. part 1. steady straining flow. Journal of fluid mechanics, 284:97-135.

[34] Mittal, R. (1999). Planar symmetry in the unsteady wake of a sphere. AIAA Journal, $37(3): 388-390$. 
[35] Mittal, R. and Iaccarino, G. (2005). Immersed boundary methods. Annu. Rev. Fluid Mech., $37: 239-261$.

[Note1] Note1. The increase of numerical errors for low Reynolds number flow past immersed boundaries is also observed by Kempe and Fröhlich [29] and Pierson and Magnaudet [43]. In their cases those are a direct consequence of IBM forcing before the implicit step of the Crank-Nicholson method. This creates an error on the forcing term that scales as $\mathcal{O}(\Delta t \mu / \rho)$.

[Note2] Note2. Single pairs of steady counter-rotating vortices are found for $\left(R e=25, \theta=75^{\circ}\right)$ and $\left(R e=125, \theta=70^{\circ}\right)$. This regime will be studied in the next section.

[Note3] Note3. We have purposely considered a range of yaw angles larger than the one discussed in the present section, since the added regimes $\left(\theta=65^{\circ}\right)$ have different wake patterns but comparable Strouhal numbers in the range $135 \leq R e \leq 160$.

[Note4] Note4. It is possible to include higher order terms, but it does not improve significantly the model. Indeed one of the drawbacks of slender body theory is that, using an expansion in $1 / \log (2 L / D)$, the expansion is not very accurate for small $L / D$ and rapidly diverges as $L / D$ goes to 0.5 Batchelor [4].

[Note5] Note5. This assumption is also expected to be valid for long cylinder $L \gg D$.

[41] Perot, J. B. (1993). An analysis of the fractional step method. Journal of Computational Physics, 108(1):51-58.

[42] Pierson, J.-L. and Magnaudet, J. (2018a). Inertial settling of a sphere through an interface. part 1. from sphere flotation to wake fragmentation. Journal of Fluid Mechanics, 835:762-807.

[43] Pierson, J.-L. and Magnaudet, J. (2018b). Inertial settling of a sphere through an interface. part 2. sphere and tail dynamics. Journal of Fluid Mechanics, 835:808-851.

[44] Popinet, S. (2015). A quadtree-adaptive multigrid solver for the Serre-Green-Naghdi equations. Journal of Computational Physics, 302:336-358.

[45] Prosperetti, A. and Tryggvason, G. (2009). Computational methods for multiphase flow. Cambridge university press.

[46] Rahmani, M. and Wachs, A. (2014). Free falling and rising of spherical and angular particles. Physics of Fluids, 26(8):083301.

[47] Ramberg, S. (1983). The effects of yaw and finite length upon the vortex wakes of stationary and vibrating circular cylinders. Journal of Fluid Mechanics, 128:81-107. 
[48] Relf, E. and Powell, C. (1917). Tests on Smooth and Stranded Wires Inclined T the Wind Direction, and a Comparison of Results on Stranded Wires in Air and Water.

[49] Ridley, J. (1982). Packing efficiency in sunflower heads. Mathematical Biosciences, 58(1):129139.

[50] Rosendahl, L. (2000). Using a multi-parameter particle shape description to predict the motion of non-spherical particle shapes in swirling flow. Applied Mathematical Modelling, 24(1):11-25.

[51] Saff, E. B. and Kuijlaars, A. B. (1997). Distributing many points on a sphere. The mathematical intelligencer, 19(1):5-11.

[52] Sakamoto, H. and Haniu, H. (1990). A study on vortex shedding from spheres in a uniform flow. ASME, Transactions, Journal of Fluids Engineering, 112:386-392.

[53] Sanjeevi, S. K. and Padding, J. T. (2017). On the orientational dependence of drag experienced by spheroids. Journal of Fluid Mechanics, 820 .

[54] Sears, W. R. (1948). The boundary layer of yawed cylinders. Journal of the aeronautical sciences.

[55] Tomboulides, A. G. and Orszag, S. A. (2000). Numerical investigation of transitional and weak turbulent flow past a sphere. Journal of Fluid Mechanics, 416:45-73.

[56] Toupoint, C., Ern, P., and Roig, V. (2018). Freely falling cylinders at moderate reynolds numbers; part 1: kinematics and wake. Journal of Fluid Mechanics (submitted).

[57] Uhlmann, M. (2005). An immersed boundary method with direct forcing for the simulation of particulate flows. Journal of Computational Physics, 209:448 - 476.

[58] Uhlmann, M. and Dušek, J. (2014). The motion of a single heavy sphere in ambient fluid: a benchmark for interface-resolved particulate flow simulations with significant relative velocities. International Journal of Multiphase Flow, 59:221-243.

[59] Vakil, A. and Green, S. I. (2009). Drag and lift coefficients of inclined finite circular cylinders at moderate reynolds numbers. Computers $\&$ Fluids, 38(9):1771-1781.

[60] Vogel, H. (1979). A better way to construct the sunflower head. Mathematical biosciences, 44(3-4):179-189.

[61] Wachs, A. (2009). A dem-dlm/fd method for direct numerical simulation of particulate flows: Sedimentation of polygonal isometric particles in a newtonian fluid with collisions. Computers E Fluids, 38(8):1608-1628. 
[62] Wachs, A. (2011). PeliGRIFF, a parallel DEM-DLM/FD direct numerical simulation tool for 3D particulate flows. Journal of Engineering Mathematics, 71(1):131-155.

[63] Wachs, A., Hammouti, A., Vinay, G., and Rahmani, M. (2015). Accuracy of finite volume/staggered grid distributed lagrange multiplier/fictitious domain simulations of particulate flows. Computers \& Fluids, 115:154-172.

[64] Wieselsberger, C. (1922). Further data on the law of liquid and air drag. Phys. Z, 23:219-224.

[65] Willmarth, W. W., Hawk, N. E., and Harvey, R. L. (1964). Steady and unsteady motions and wakes of freely falling disks. Physics of Fluids (1958-1988), 7(2):197-208.

[66] Zastawny, M., Mallouppas, G., Zhao, F., and Van Wachem, B. (2012). Derivation of drag and lift force and torque coefficients for non-spherical particles in flows. International Journal of Multiphase Flow, 39:227-239.

[67] Zdravkovich, M., Brand, V., Mathew, G., and Weston, A. (1989). Flow past short circular cylinders with two free ends. Journal of fluid mechanics, 203:557-575.

[68] Zdravkovich, M. M. (2003). Flow around Circular Cylinders: Volume 2: Applications, volume 2. Oxford university press.

[69] Zhao, M., Cheng, L., and Zhou, T. (2009). Direct numerical simulation of three-dimensional flow past a yawed circular cylinder of infinite length. Journal of Fluids and Structures, $25(5): 831-847$. 